\section{THE ENERGETICS OF ORDERED INTERMETALLIC ALLOYS (OF THE TRANSITION METALS)}

\author{
R. F.. Watson, ${ }^{1}$ M. Weinert, ${ }^{1}$ J. W. Davenport, ${ }^{1}$ \\ G. W. Fernando, ${ }^{2}$ and L. H. Bennett ${ }^{3}$ \\ ${ }^{1}$ Brookhaven National Laboratory, \\ Department of Physics, Upton, New York 11973 \\ University of Connecticut, \\ Department of Physics, Storrs, CT 06269-3046 \\ ${ }^{3}$ National Institute of Standards and Technology, \\ Metallurgy Division, Gaithersburg, MD 20899
}

\section{INTRODUCTION}

Theory and experiment are both useful in the development of new scientifically interesting and technologically relevent materials. On the theory front, one would like to predict the occurrence of stable and metastable phases as well as gaining insights into the chemical bonding which underlies the phase formation. In addition, one would like to explore the mechanical, electronic, optical and other properties of the phases in question. One of the first steps in doing this is to explore the atomically ordered phases and it will be the ordered transition metal ailoys which will be of
concern of this chapter.

When estimating phase stability, there is almost a $10^{10}$ variation in computational effort which may be applied. This ranget, on the upper end, from "all bells and whistles" efforts to solve Schrodinger's equation with some rigor for a solid to a lower end involving schemes which can be implemented, by hand, on a piece of paper if one has maintained one's elementary arithmetic skills. Both ends of the spectrum if one their uses and both will be touched on in this chapter - we will consider structura maps and model schemes, such as Miedema's, on one hand and, on the other, we will inspect the results of full local density potential augmented basis set calculations. Augmented basis set calculations are the most expensive, computationally, of the conventional band structure schemes but, in our view, offer the most reliable treatment for transition metal $d$ (and actinide $f$ ) bands. Being expensive, they should not be casually done for large numbers of cases. However, tiney may be deemed to be henchmarks with respect to which other schemes may be measured or from which the parameters employed in other schemes may be derived.

Figure 1 is an example of the product from a set of estimates of heats of formation for ordered phases, in this case in the Pt-Ti system. We will have occasion to

\section{DISCLAIMER}

This report was prepared as an account of work sponsored by an agency of the United States Government. Neither the United States Gnvernment nor any agency thereof, nor any of their employees, makes any warranty, express or implied, or assumes any legal liability or responsibility for the accuracy, completeness, or usefulness of any information, apparatus, product, or process disclosed, or represents that its use would not infringe privately owned rights. Reference herein to any specific commercial product, process, or service by trade name, trademark, manufacturer, or otherwise does not necessarily constitute or imply its endorsement, recommendation, or favoring by the United States Government or any agency thereof. The views and opinions of authors expressed herein do not necessarily state or reflact those of the United States Government or any agency thereof.

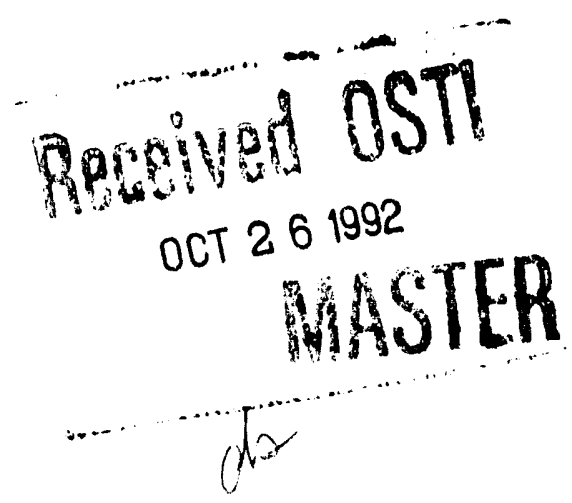


ceturn to this case later, but we might note several features of the system and of the calculations here. First, the 50:50 phase undergoes a Martensitic phase transition at the temperature of $\sim 1000^{\circ} \mathrm{C}$, a higher temperature than that at which such transitions

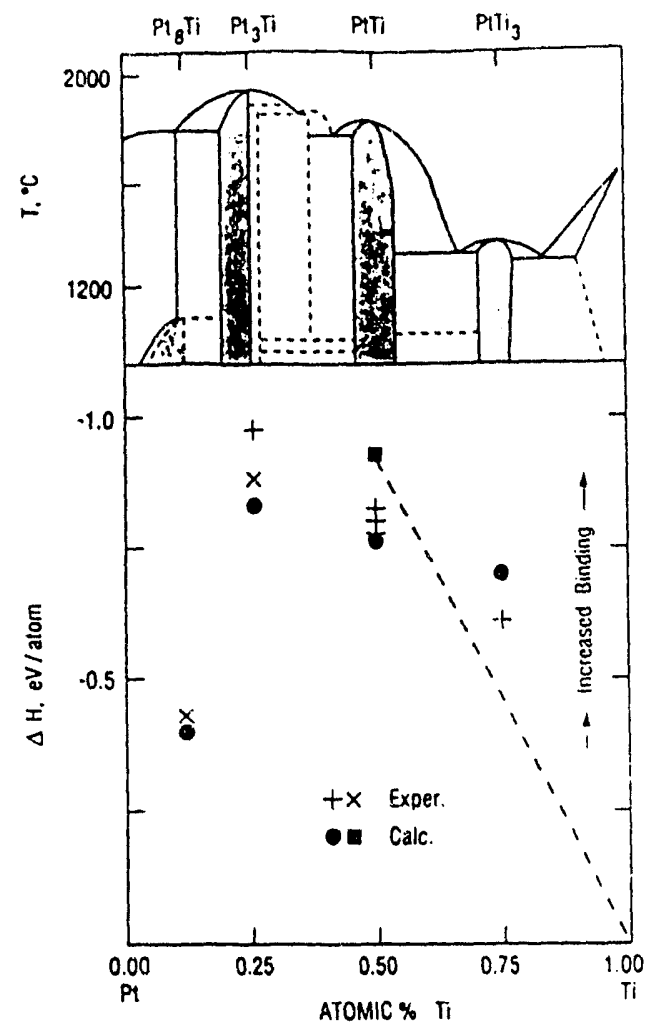

Figure 1. Calculated and experimentally determined beat of formation for $P t_{b} T_{i}, P_{1} T_{i}\left(C u_{3} A u\right.$ structure), PITi (AuCd and CACl structurea) and PtTis (AIS). The upper panel shows a rough sketching of the Pt-Ti phaee diagram with the ahaded regions being the phases represented by the calculations. The cromes indicate ealorimetric mensurements and the $x$ 's emf meanuremente At 50:50 concentration, the equare indicaten the calculated beat for the low vemperature $A u C d$ structure with the bith temperature $\mathrm{CACl}$ atructure phese, the circle, correctly shown to be leat bound. The dached line is the phase diegrum at $\sim 1000^{\circ} \mathrm{C}$ for the 50:50 phree is the Martensitic tranaition between these two pheres. The calculated heats were obtuined with relativistic full potential LDA calculations with spin-orbit effects included in the valence bands.

commonly occur (this is represented by the dashed line in the phase diagram at $\sim 50: 50$ ). As for the calculations, the calculated and experimental heats of formation, $\Delta H$, apree to within the scatter in experiment, i.e. $\sim 1 / 10 \mathrm{eV} /$ atom. The $\Delta H$, like the phase diagram above, display a strong skewing towards the strongest alloy formation occurring for the $P t$-rich systems. In general one expects the strongest compound 
formation to occur at 50:50 composition because this lets one have the maximum number of unlike atom nearest-neighbor pairs, hence the maximum number of bonds favoring alloy formation. As will be discussed later, $d$ band bonding is responsible for the skewing. The fact that the calculated $\Delta H$ for $P t T_{i j}$ lies above the line drawn between the $\Delta H$ for $P_{t} T_{i}$ and the zero for $T_{i}$ indicates that $P_{t} T_{i j}$ is stable relative to a two-phase mix of PtTi and $T i$ baving the same overall composition. Similarly, $P t T i$, $P_{t_{3}} T_{i}$, and $P_{t_{3}} T_{i}$ are stable against competition from two-phase mixes of adjacent phases. Thus the calculatal (as well as the experimental) $\Delta H$ are consistent with essential features of the phace diagram. The $P t-T i$ system offers a particular test of one's ability to calculate as 'will be discuseed towards the end of this chapter.

This chapter will concentrate on the following:

- Phyrical Parameters Controlling Phase Stability

$\rightarrow$ Hume-Rothery $\rightarrow$ Miedema Hamiltonian

$\rightarrow$ Structural Maps

- Wave Functions (Multi \& Single Electron)

$\rightarrow$ Potentials (Chodorow and Local Density)

$\rightarrow$ Total Energies $\rightarrow$ Details (Such as $k$ Sets)

- A Comment on Entropy Terms

- Cohesive Energies $\rightarrow$ Electron Promotion Energies

$\rightarrow$ Multiplet Effects with Local Density

$\rightarrow$ Issues Associated with Dealing with Hund's Rule Orbital Effects

- Structural Energies of the Elemental Solids

- Energies Associated with Atomic Positions Deviating from Perfect Lattices

- Charge Transfer - A Measure of "Chemical" Effects $\rightarrow$ Charge Tailing

- Heats of Formation of Ordered Compounds

$\rightarrow$ Bonding Trends as Evidenced by Inspection of Densities of States

\section{HUME-ROTHERY, STRUCTURAL MAPS, AND SIMPLE HAMUTONIANS}

As Hume-Rothery emphasized, many allojing trends may be understood in terms of electron to atom ratios (i.e. bandfilling), of the relative sizes of the constituents and in the strength of bonding as measured, say, by an electronegativity difference. For example, substitutional alloying occurs most readily when the atoms are similar in size and when the bonding (i.e. the difference in electronegativities) is not too great, since strong bonding encourages the formation of ordered compounds rather than a substitutional alloy. Size has taken on different guises over the years: in metallic systems the useful measure would appear to be actually size as measured by atomic valume or radius while for covalently bound systems it is common to employ the ion core radius, that is the repulsive region of the pseudopotential, as a measure of effective size.

Otber factors have been invoked in addition to Hume-Rothery's three. For example, the electron density in the outer reaches of an atomic cell is featured in Miedema's Hamiltonian ${ }^{1}$ while the relative strengths of $s$ versus $p$ bonding arise in considerations of covalent bonding. Discussions of transition metal bonding often esnploy the position of the $d$ band centers of gravity and their band widths as relevant parameters.

Electronegativity differences have been the subject of much curiosity because not only are they presumed to provide a measure of bonding strength, but also an indication of the direction and magnitude of charge transfer. There are almost as many electronegativity scales as there are workers who have considered the issue. 
Une is the Mulliken scale which is based on the ionization energies and electrom affinities of the free atoms, that is the propensity of an atom to keep the electrons it has, as well as the tendency to gain another. This is intuitively a most attractive definition, but unfortunately it is obtained for free atoms rather than for atoms actively involved in bonding. The Pauling and Phillips scales involve the energetics of bonding and the dielectric properties of solids respectively. There are a number of "metallurgical" scales where the electronegativity has been taken proportional to an element's chemical potential as manifested by its work function. Such a scale has been cmployed' by Miedema in his model Hamiltonian. These and other electronegativity scales are in general agreement that the electronegativities increase as one traverses the transition metals from left to right, that is, a left hand member such as $T_{i}$ loses charge to $N i$ which is on its right. There are, however, differences in detail. For example, Pauling has Au the most electronegative of the metals whereas work functions indicate $R e, O_{s}, I r$, and $P t$ to be more electronegative than Au. This issue will be returned to later.

In addition to size and electronegativity, the third Hume-Rothery parameter is band-filling. The crystal structures of the transition metals, when nonmagnetic, occu in the sequence indicated in Figure 2. This trend has been rationalized by the tight

\begin{tabular}{|c|c|c|c|c|c|c|c|c|}
\hline $\mathrm{Sc}$ & $\mathrm{Ti}$ & $V$ & $\mathrm{Cr}$ & $\mathrm{Mn}$ & $\mathrm{Fe}$ & Co & $\mathrm{Ni}$ & $\mathrm{Cu}$ \\
\hline$Y$ & $\mathrm{Zr}$ & $\mathrm{Nb}$ & Mo & $\mathrm{Tc}$ & Ru & $\mathrm{Rh}$ & $\mathrm{Pd}$ & $\mathrm{Ag}$ \\
\hline La & $\mathrm{Hf}$ & $\mathrm{Ta}$ & W & $\mathrm{Re}$ & Os & Ir & $\mathrm{Pt}$ & $A u$ \\
\hline
\end{tabular}

tcp

$$
A 15\left(C r_{3} S i\right) \rightarrow \sigma \rightarrow\left\{\begin{array}{l}
\alpha M n \\
\mu\left(F e_{7} W_{6}\right)
\end{array}\right.
$$

Figure 2. The sequence of structures which arise as a function of $d$ band filling as the transition metals are traversed, providing that the metals, in question, are constrained to be nonmagnetic. The same sequence describes the alloys as a function of band filling, providing there is the insertion for the occurrence of the topologically clowe-packed, tep phuses, and providing that chemical bonding is not too strong ( 10 as to encourage ordered compounds) and the relative sires of the alloy constituents are favorable. If several tep phases occur, they occur in the sequence shown in the bottom panel of the figure.

binding estimates of Pettifor ${ }^{2}$ and the work of otbers. This trend also describes transition metal alioy formetion providing one inserts a group of topologically-closepacked $(t c p)$ or Frank-Kusper phases as indicated. These include the $\sigma\left(C r F_{c}\right)$, the $\mathrm{A} 15$, and the $\mu\left(F_{e} W_{6}\right)$ structures, as well as the $\alpha M n$ structure which has packing ${ }^{3}$ close to that of the tcp phases. The tcp are alloy phases having different atomic sites of differing atomic volume involving the nonplanar packing of atoms having 12-, 14-, 15-, and 16-fold near-neighbor coordinations. The nonplanar packing makes these phases brittle. 
An exanple of where such simple band-filling considerations control alloy phase behavior is to be seen for the MoIr system in Figure 3. bce Mo is followed by A15, $\sigma$, and $h c p$ intermediate phases, finally ending in substitutional $I r$-rich $f c c$ alloys. Important to this trend is the absence of strong compound formation which would encourage the occurrence of highly ordered line compounds.

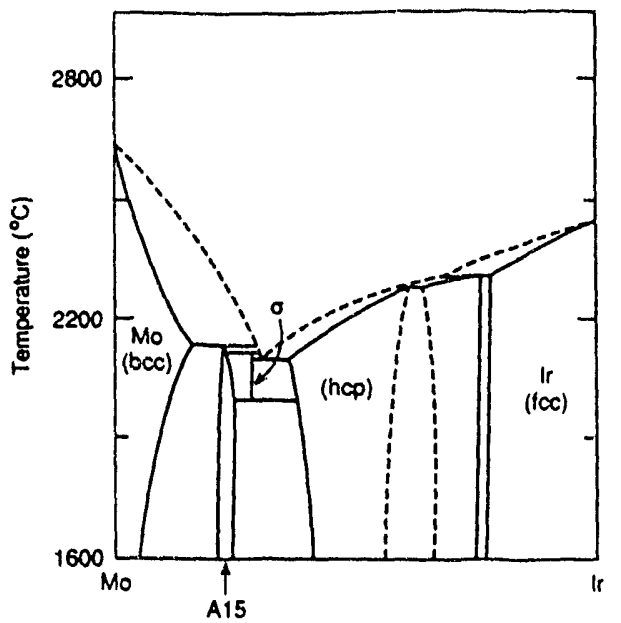

Figure 3. A sketch of the Mo-Ir phase diagram showing the sequence of structures bce $\rightarrow A 15 \rightarrow$ $\sigma \rightarrow h c p \rightarrow f c c$, as a lunction of band filling, indicaled in Figure 2.

The 12-fold coordinated sites of the tcp phases, involve an icosahedron, normaily a somewhat distorted iscosahedron, of nearest neighbors. In contrast, the 14-, 15-, and 16-fold sites involve 2,3, and 4 close lying, "major bonding" nearest neighbors respectively. The two of the 14-fold lie in a line, squashing the atom's environment along the line as in the linear chains of atoms in the A15 phases. The 3-fold are arranged coplanarly while the 4 -fold are (roughly) tetrahedral. These major bonds or disclinations form nets through the crystal lattices.

There is another class of crystal structures involving the environments Bernal encountered ${ }^{5,6}$ in his glass constructs. These bave 10-, 9-, and 8-fold environments involving 2, 3, and 4 minor bond lines respectively which are arranged at atomic sites much like the major bonds of the tcp systems. However, these are associated with distant rather than near-lying nearest neighbors. Both classes of structures figure importantly in transition metal alloy formation and they feature ill-packed local atomic environments in contrast with those of the well-packed $f c c, h c p$, and bcc: phases.

The tcp and closely related phases fall into two groups:

There are those with a majority of small atom sites. These include the Laves phases (having just 12- and 16-fold sites), the hard magnets such as $\mathrm{SmCos}_{\mathrm{s}}$ $N d_{2} F e_{14} B$, and the kydrogen storer $L a N i_{5}$. These systems generally form at close to ideal stoichiometry. 
Then there are those phases without a majority of such 12 fold sites. These include the $c 1160$ and cll62 which are related to icosaliedral phatse systems such as . $4 / .9$ $M g_{32}, A l_{5} C u L i_{3}$. Then there are $A 15, \sigma(C r F e)$, and $\lambda$, and finally we have $\alpha M M_{i}, c$ $\mathrm{Mg}_{23} A l_{30}$ whose packings do not conforn strictly to the tcp construct. These alloys, when involving transition metals, occur at the favored $d$ band occupancies indicated in Figure 2 sometimes over significant concentration ranges (at the cost of mixed atom occupancies on some sites). Significant concentration ranges are encouraged by the strurtures having distributions (not just 12- and 16-fold) in sites, and are most likely to occur ${ }^{7}$ when the volumes of the constituent atoms differ by twenty to forty percent. Apparently this lets the systems make "best use" of the differing atomic sites.

We could have plotted a "structural map" with $d$ electron count as one coordinate and volume ratio as another. Better yet, one could add a third coordinate which is a measure of strength of bonding, such as an electronegativity difference. This would show that providing: the relative volumes are alright and the chemical bonding isn't too strong, these tcp phases will occur at the suitable band filling. Such considerations may be extended to transition metal-rich systems having modest amounts ${ }^{8}$ of $A l, S i$ Ge, etc. present.

A structural map for the ordered 50:50 transition metal alloys appears in Figure 4. One coordinate is band filling as measured in terms of $d$ band vacancies. This was done because it is easier to define the top, rather than the bottom of the $d$ bands, and hence easier to count in terms of $d$-band holes. The second coordinate is a transition metal electronegativity scale ${ }^{9}$ based on the propensity of the $d$ bands to gain or lose $d$ count through hybridization. The scale is consistent with work function scales in that the electronegativities of the noble metals are less than those of their immediate transition metal neighbors. The map shows a $t c p \sigma$ phase region of small binding, i.e. small electronegativity differences, centered at an electron vacancy count of four. To the right are $f c c$ based phases and to the left $b c c$ ones, consistent with band filling expectations. At large binding, there is a wedge of $B C r$ structure phases. This structure involves atomic sites having Bernal environments which, as discussed above, are of low symmetry and low atomic coordination - lower atomic coordination than might normally be expected for metallic systems. The separation of the various structures in the map is fairly good. What is of particular interest are the clear errors. $M n P d, F e R h$, and $F e C o$ are $b c c$ in the $f c c$ region and this, presumably, follows from their magnetism, just as ferromagnetic $F e$ is bcc though if nonnagnetic it should be close-packed (see Figure 2). CuPd also is on a bcc lattice though this occurs at a stoichiometry closer to $C u_{3} P d_{2}$. Whether on or off 50:50 it is surprising that this system orders in this way. The remaining major "error" consists of $\operatorname{Ir} T i, R h T i$, and $R u T a$ which are normally listed as in the $f c c$-based $C u A u I$ structure which is related to the $\mathrm{CsCl}$ structure by a tetragonal distortion. Inspection of the $\mathrm{c} / \mathrm{a}$ ratios for the three systems in question shows values close to that of the cubic bcc and not the cubic $f c c$ structure. In other words, these CuAuI systems lie on bcc lattices, admittedly tetragonally distorted ones, consistent with their presence in the bcc region of the structural map.

Villars has gathered the most recent and complete compilation of crystallographic data. ${ }^{10}$ Using his data base of observed crystallographic structures, he has searched" for the "best choices" of coordinates for structural mapping. The search emphasized structures encountered in semiconductor compounds and this is reflected in the result. For example size is represented by an ion core size rather than by atomic volume which might be more appropriate to metallic binding. Phillips, Rabe, and Villars have used $^{12,13}$ these coordinates to define regions favorable to the formation of: icosahedral, high $T_{c}$ superconductor, and high $T_{c}$ ferroelectric phases. These mappings appear to provide a useful first cut in the search for new systems. 
Structural maps may provide useful qualitative estimates concermag the occurrence of hitherto undetected phases and the basis for challenging purportedly observed phases. Such maps are a lot cheaper than the calculations reported clscwhere in these proceedings. Of course, such Hume-Rothery type parameters may be cmployed in effective Hamiltonians and one most successful of such endeavors is the subject of the next section.

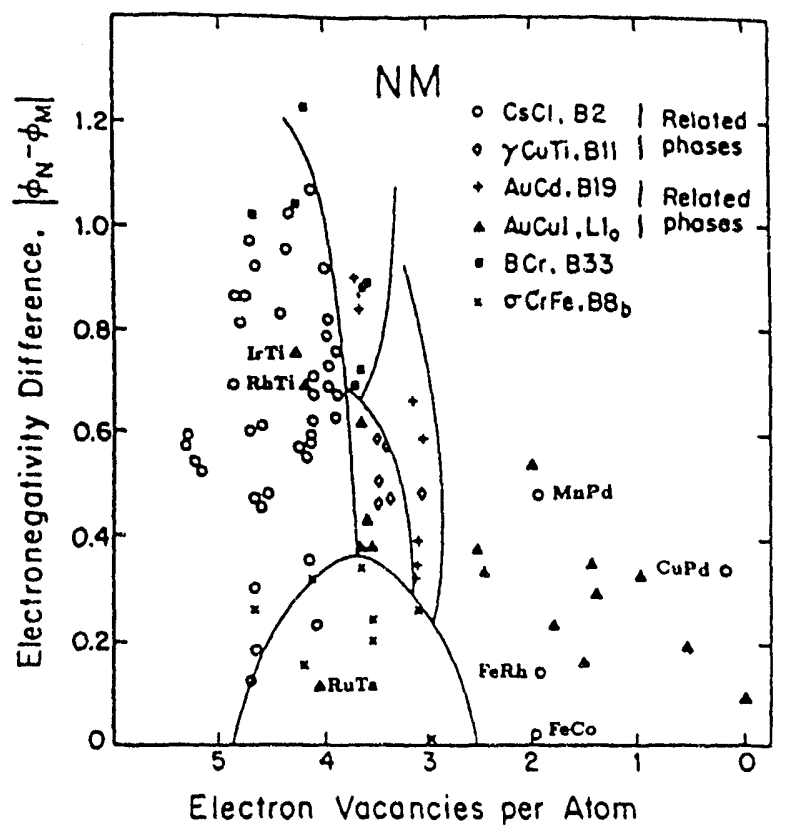

Figure 4. A structural map for the 50:50 transition metal-transition metal alloys where one coordinake is the difference in electronegativities which were derived, based on the propensity for a band structure's $d$ band to gain or loee charge through hybridization. The second coordinate is a measure of the aloy's $d$ band occupation as indicated by the average number of unoccupied $d$ band levelo appropriate to the two elemental alloy constituents. The labeled points are "errors" in the map which are diecuened in the text.

\section{THE MIEDEMA HAMILTONIAN}

Miedema employed a cellular approach where the electron density, $n$, is derived from the elemental bulk modulus. In the scheme, there is an energy cost in bringing unlike atoms $A$ and $B$ to a common density at their mutual boundary $+\alpha\left(n_{A}^{1 / 3}-n_{B}^{1 / 3}\right)^{2}$ where $\alpha$ is a positive constant. To this term is added a bonding energy $-\beta\left(\phi_{A}-\right.$ $\left.\phi_{B}\right)^{2}$ where $\beta$ again is a positive constant, and the $\phi$ 's are chemical potentials or electronegativities besed on work functions. 
The binding energy' is then

$$
E \propto \alpha\left(n_{A}^{1 / 3}-n_{B}^{1 / 3}\right)^{2}-\beta\left(\phi_{A}-\phi_{B}\right)^{2}+\gamma
$$

where the negative term $\gamma$ occurs when one element is a transition metal and the other is not and its value depends upon which column in the periodic table the latter comes from.
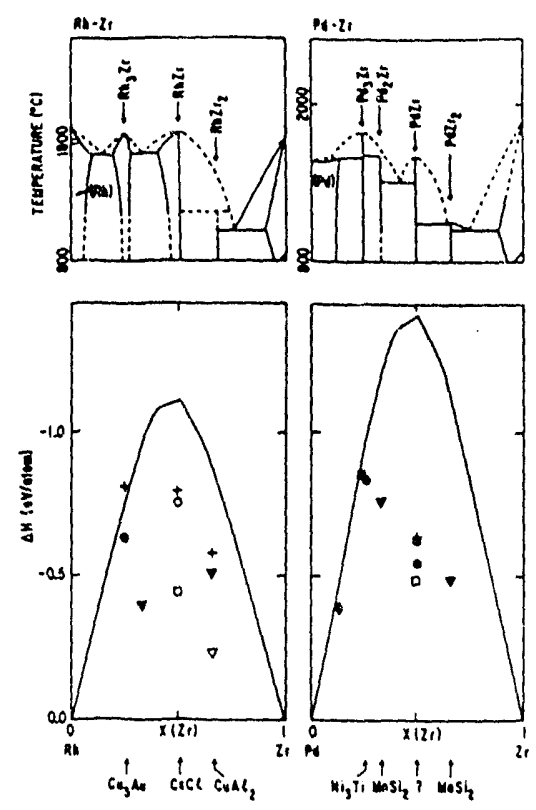

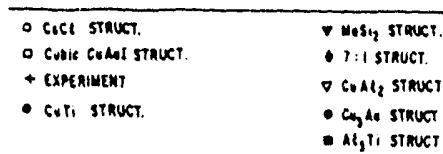

Figure 5. Heats of formation and phase diagrame for the Rh-Zr and $P d-Z r$ ayatems. Below the lower panel are indicated the atructures in which the observed ordered phasea occur. The plotted points are based on muffin-tin potential semi-relativistic LDA calculations, while the solid lines are Miedema's prediction as a function of composition.

With different prefactors

$$
E=\delta\left\{\alpha\left(n_{A}^{1 / 3}-n_{B}^{1 / 3}\right)^{2}-\beta\left(\phi_{A}-\phi_{B}\right)^{2}+\gamma\right\}
$$

works well for bulk solids, surfaces, diatomic inolecules and (with minor rewriting) interstitial ions. 
Considering the quality of results versus the cust of calculating then: Miedema's scheme is a "Best Buy." The predictions are by no means perfect as can be seen in Figure 5. The overall scale of the Miedema results is off for Pd-Zr but this is one of the worst cases which could have been chosen. Generally the Miculema scheme yields very reasonable results for the 50:50 compounds. There is a pronounced skewing of the phase diagrams and of the heats of formation towards $R h$ and $P d$, much as has already been seen in Figure 1. Miedema's heats do not depend on crystal structure and the concentration dependence appears in a volume dependent prefactor associated with how much surface of atom $A$ is there to be brought into register with how much of $B^{\prime}$. This does not yield the scale of the skewing seen here and often it predicts skewing in the wrong direction (a band theory explanation for this skewing will appear at the end of this chapter). Nevertheless, Miedema's estimates, like structurial maps, can provide invaluable first estimates concerning the occurrence and energetics of alloy phases.

\section{-BAND THEORY}

Modern band structure methods make use of the local density approximation to reduce the many electron problem to a one-electron Hamiltonian. Normally, we fill one-electron states up to a Fermi level (perhaps with partial occupations in its vicinity). The result is a single product, if you will a single-determinant, wave function. Not only does this omit correlation effects important to heavy fermion and high $T_{c}$ superconducting systems, but it does not have the multi-determinant character essential to the description of Hund's Rule effects in atoms.

One class of one-electron basis functions are augmented basis functions, where an analytic basis is employed in the interstitial region, "augmented" by explicit solutions of Schrodinger's (or Dirac's) equations within atomic spheres at each of the atomic sites. A ugmented basis set calculations of this type are the safest for transition metals, albeit the most expensive computationally. Relativistic calculations of this class are relied on in these talks.

Most of the calculations of this chapter involve the linearized augmented Slatertype orbital (LASTO) approach "where we have Bloch functions

$$
\psi_{N L}(\vec{r})=\frac{1}{\sqrt{N_{c}}} \sum_{\vec{R}} \mathrm{e}^{i \vec{E} \cdot \vec{R}_{\phi_{n \ell m}}(\vec{r}-\vec{R})}
$$

constructed from Slater-type orbitals in the interstitial

$$
\phi_{n \ell m}(\vec{r})=r^{n-1} e^{-\xi r} Y_{\ell m}(\hat{r})
$$

augmented in atomic spheres

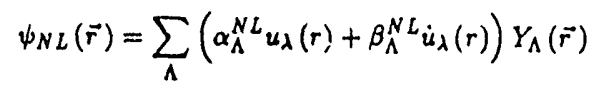

where $u(r)$ is the solution of the radial equation in the atomic sphere, $\dot{u}(r)$ is its energy derivative (and is orthogonal to it) and $\alpha$ and $\beta$ are weights determined by matching to the wave function outside. Using two $s$, two $p$, two $d$, and a single $f$ Slater-type orbital per site in the interstitial yields band results equivalent to an LAPW calculation but with a $25 \times 25$ matrix, per site, to be constructed and diagonalized. Common basis sets and common atomic spheres will be used for a compound and for the elemental solids whose total energies will be compared with that of the compound.

Local density potentials, as we now know them, did not exist in the early days of band theory. One early potential is Chodorow's for copper. It is based on a 
solid's version of atomic Hartree-Fock theory - what might be called a Wigner-SeitzHartree-Fock-VanVleck-Hubbard model in hindsight. There are lessons to be learnt from this class of potential and granted this, we'll make a side-trip to atomic HartreeFock theory.

Consider two-electron matrix elements between orbitals

$$
\begin{aligned}
& \phi_{a}(\vec{r}) \equiv P_{a}(r)^{a} Y_{l}^{m}(\theta, \phi) X_{l}^{a}(m,) \\
& \text { with } \frac{1}{\mid \overrightarrow{r_{12} \mid}}=\sum_{k} \frac{r_{k}^{k}}{r_{>}^{k+1}} P_{k}(\cos \omega) \\
& =\sum_{k} \frac{r_{<}^{k}}{r_{>}^{k+1}} \cdot \frac{4 \pi}{2 k+1} \sum_{m} Y_{k}^{m}\left(\theta_{1}, \phi_{1}\right) Y_{k}^{m}\left[\left(\theta_{2}, \phi_{2}\right)\right]^{\circ} \\
& \text { then } \\
& \iint \phi_{a}^{*}\left(\vec{r}_{1}\right) \phi_{b}^{*}\left(\vec{r}_{2}\right) \frac{1}{r_{12}} \phi_{c}\left(r_{1}\right) \phi_{d}\left(r_{2}\right) d r_{1} d r_{2} \\
& =\delta\left(m_{d}^{d} m_{l}^{b}\right) \delta\left(m_{\ell}^{c} m_{\ell}^{a}\right) \delta\left(m_{\ell}^{a}+m_{\ell}^{b}, m_{\ell}^{c}+m_{l}^{d}\right) \\
& \sum_{k}\left\{c^{k}\left(\ell_{a} m_{a}, \ell_{c} m_{c}\right) c^{k}\left(\ell_{d} m_{d}, \ell_{b} m_{b}\right)\right\} \\
& R^{k}(a b c d)
\end{aligned}
$$

where the $c^{k}$ are angular integrals, which apart from a $\sqrt{\frac{1 x}{2 t+1}}$ are Gaunt coefficients, and

$$
R^{k}(a b c d)=\iint \frac{r_{c}^{k}}{r_{>}^{k_{1}}} P_{a}\left(r_{1}\right) P_{b}\left(r_{2}\right) P_{c}\left(r_{1}\right) P_{d}\left(r_{2}\right) d r_{1} d r_{2} .
$$

Coulomb terms involve the interaction of a density, $\left|\phi_{a}\right|^{2}$, interacting with another, $\left|\phi_{b}\right|^{2}$

$$
\begin{aligned}
\text { The interaction } & =\sum_{k} \text { angular terms } \cdot R^{k}(a b, a b) \\
& \equiv \sum_{k} \text { angular terms } \cdot F^{k}(a b) .
\end{aligned}
$$

Exchange involves the interaction of an overlap density $\phi_{a}^{*} \phi_{b}$ with its complex conjugate $\phi_{a} \phi_{b}$

$$
\begin{aligned}
\text { The interaction } & =\sum_{k} \text { angular terms } \cdot R^{k}(a b, b a) \\
& \equiv \sum_{k} \text { anguiar terms } \cdot G^{k}(a b) .
\end{aligned}
$$

For electrons in the same shell with the same radial function $P(r), F^{k}(a a)=$ $G^{k}(a a)$.

In the Hartree-Fock description of an atomic shell of $n$ electrons there will be $n$ spherical Coulomb $F^{0}(a a)$ minus one self (Coulomb) exchange $F^{0}(a a)$ in the potential sampled by one electron. This leads to a $\frac{n(n-1)}{2} F^{0}(a a)$ term in the total energy of the system. This $F^{0}$ is the unscreened $U$ of Hubbard and Anderson Hamiltonians and the "relative" of the exchange-correlation hole in local density theory.

The aspherical $F^{k}$ and $G^{k}$ terms underly multiplet theory for the atoms and aspherical effects in the solids.

A Hartree-Fock derivation for a solid with a valence shell having $n$ electrons per atom in that shell, yields a $(n-x) F^{0}$ contribution to the one electron energy (or 
potential), where $x$ depends on the filling of the valance band (i.e., $x \rightarrow 1$ when the band is full). There is a full self-Coulomb hole, but the extent to which it is localized on a single site, involving $F^{\circ}$ alone, depends on band filling.

Taking a $d^{10} s \mathrm{Cu}$ atomic wave function, Chodorow defined a crystal potential with $9 d+$ is Coulomb terms (i.e., a full $d$ hole was "localized" on the site) plus interelectronic $k \neq 0 \quad F^{k}$ and $G^{k}$ terms. The "full potential" local density and the Chodorow's Slater-Condon-Racah approach treat the aspherical coulomb testns identically. The differences arise in the aspherical (and spherical) exchange-correlation terms. A computaticanal shortcoming of a scheme, such as Chodorow's, is in its sampling of one-electron wave functions - not just electron densities. Apart from a possible screening of the $F^{k}$ integrals, it also neglects correlation but unlike local density theory it allows the treatment of spin and orbital magnetic effects on an equal basis, a matter which will be returned to later.

The local density approximation has been defined in detail elsewhere in these proceedings and this will not be repeated here. One feature of this class of potential is that it has its aelf-Coulomb hole centered on the point at which the electron is sampling the potential. A spin-dependent local density (LSDA) cinss of potential has been derived where there are different exchange - coorelation terms for electrons of different $M_{1}$. This allows spin-polarized atomic and band structure calculations to be performed.

Perdew and others have worked ${ }^{15}$ on gradient corrections to the LDA potential. As evidenced by energies obtained for atoms, a satisfying gradient correction may now exist. Gradient corrections have not been employed in the calculations reported on in this chapter. They are expected to be numerically important to cohesive energy estimates, but otherwise to have little qualitative effect on the results to be discussed. (We also note that according to the Ames-Iowa group, these gradient corrections have problems when dealing with the $5 d$ elemental solids.)

Until recently, it has been common to employ "muffin-tin" potentials which are limited to spherical terms at atomic sites and constant terms in the interstitial region. Now, "full" potentials which are "without shape approximations" are being increasingly employed. These account for the aspherical character of the charge density, hence the potential, throughout space. There is a substantial, i.e. order of magnitude, increase in computing cost on going from the muffin-tin to the full potential, but this is essential when obtaining energies for systems having low-site symmetries such as the Frank-Kasper phases or phases where the atoms have Bernal environments.

While we calculate total energies with our band calculations, we are concerned with calculating differences between total energies and not with absolute values of individual total energies. This is essential to our success. For example, we are concerned with the difference between an element in the $b c c$ versus the $f c c$ structure

$$
E_{b c c}-E_{f(c)}
$$

or a cohesive energy

or a heat of formation

$$
H_{\text {coh }}=E_{\text {solid }}-E_{\text {free atom }}
$$

$$
\Delta H=E_{\text {composind }}-\sum_{i} x_{i} E_{i}
$$

where $x_{i}$ is the fraction of element $i$ in the compound, or the competition between the $\Delta H$ of one phase with that of another or a combination of others. The success or failure of such enterprises then resides in our ability to calculate diverse total energies to a common accuracy. 
In doing this, sufficient care snust, of course, be taken with such matters as cheloice of $\vec{k}$ sets, of cut-offs in reciprocal space and in the common dioice of basis sets of sufficient variational freedom.

\section{A COMMENT CONCERNING ENTROPY}

It is normal to limit consideration of entropy terms to those of configuration entropy (atomic mixing) and to vibrational entropy when dealing with an alloy system.

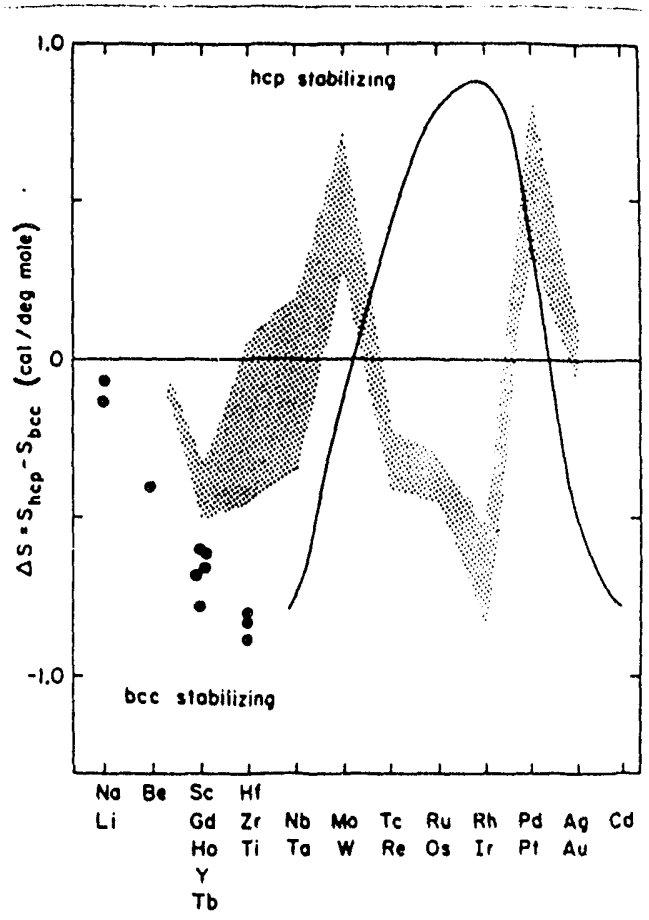

Figure 6. Comparison of the electronic contributions to the elemental bcc-hcp entropy differences with the experimental valuea (the circles) for the lefthand elements and with Kaufman and Bernstein's CALPBAD estimates (the solid line) for the heavier transition elements, chosen so as to allow the observed phase diagrams to be reproduced. The shaded region indicate the calculated electronic terma, the apread being due to the spread in exiating band-theory preductions of $n(c r)$

\section{There is a term associated with electronic excitations, namely}

$$
S_{e l}=\frac{1}{3} \pi^{2} n\left(\epsilon_{F}\right) k_{B}^{2} T+\text { higher orders }
$$

which is normally neglected since the electronic specific heat of a solid is swamped by the vibrational entropy. As Grimvall pointed out, ${ }^{16} S_{e l}$ may be small but the change in $S_{e l}$ in going from one structure to another need not be inconsequential. This 


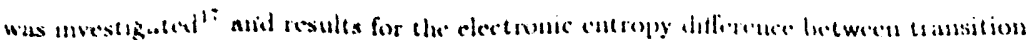
metals in the bic and in the for structure appear in Figure 6 . These are compared with the cosal change in entrupy as observed experimentally for the left hand of the transition metal row and in Kaufinan and Bernstein's CALPHAD estimates ${ }^{18}$ for the remainder for which there are no $f a r$ bec phase transitions to be observed. The ekesranic terms are of the under of the latal which involves combined electronic plus vibrational terms. This suggests that if vibrational cutropy changes are to be accounted for in an alloy calculation, then the electronic terms should also be considered as well. This is trivial to do if the densities of states have bien obtained for the systems in question.

Martensitic transitions are of concern elsewhere in this volume. These involve the transition from a higher symmetry high temperature phase to a lower symmetry low temperature one. Going to lower symmetry will break the degeneracies of orbital levels and if some of these are in the vicinity of the Fermi level, the splitting leaving some below, and some above ef, will enhance the binding energ of the low temperature phase. Concomitant to this will be a lowering of $n(\epsilon F)$, hence $S_{e l}$. A mesurable fraction of the entropy change, stabilizing the high temperature phuse, can be expectet to come from such a electronic term.

\section{COHESION OF THE ELEMENTAL METALS}

The cobesive energy of an elemental solid is the total energy of the solid minus that of the atom, namely

$$
\Delta H_{\text {ooh }}=E_{\text {solid }}-\bar{E}_{\text {etom }} \text {. }
$$

With or without gradient corrections, there is the question of to what extent LDA treats the solid and the free atom equivalently. There is another complication. An experimental $\Delta H_{\text {coh }}$ is measured with respert to the lowest lying multiplet level of the free atom, while an LDA or LSDA calculation for an atom is usually done for the average over a number of multiplet states. In the case of the LSDA, this might be over a set of states of maximum $M$, for the atomic configuration in question, i.e. states associated with the maximum allowable spin multiplicity. A common practice is to do calculations for different atomic configurations and to define the case having the lowest energy as the LSDA "ground state." This average over multiplet levels may not include the actual ground level of the atom. One possibility is to use experimental data to define the promotion energy from the actual ground state to the multiplet average in question.

The corrected heat is then

$$
\Delta H_{\text {coh }}=E_{\text {solid }}-\left(E_{\text {ctom }}-\Delta_{p}\right)
$$

wher: $\Delta$, is the promotion energy, determined from experiment, from the atom's ground state to the center of gravity of configuration that the calculated $E_{\text {atom }}$ corresponds to.

Results obtained in the two ways appear in Figure 7 for the $5 d$ metals. Correcting the atomic reaults $s 0$ that reference is made to the actual atomic ground state

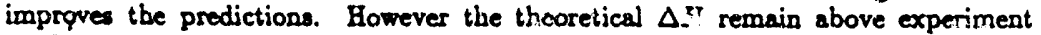
implying that local dessity theory has done a poorer job describing the free atom than the solid. Corrected results for the $d^{n}, d^{n-1} s$, and $d^{m-2} s^{2}$. configurations for the $4 d$ elements appear in Figure 8 (there is insufficient $d^{m}$ spectral data for the $5 d^{\prime}$ 's to make such a comparison for them). Seeing the change on going from $d^{n-2} s^{2}$ to $d^{n-1} s$, it is surprising that there is no further change on going to $d^{n}$. Using ${ }^{19}$ other reference configurations, e.g. $d^{n-1} p$, yields results which fall within the envelope of 
the predictions seen here. This discrepancy between theory and experiment is one of the most glaring shortcomings of local density theory. It remains to be seen whether, and to what extent, gradient corrections to LDA improves on this.

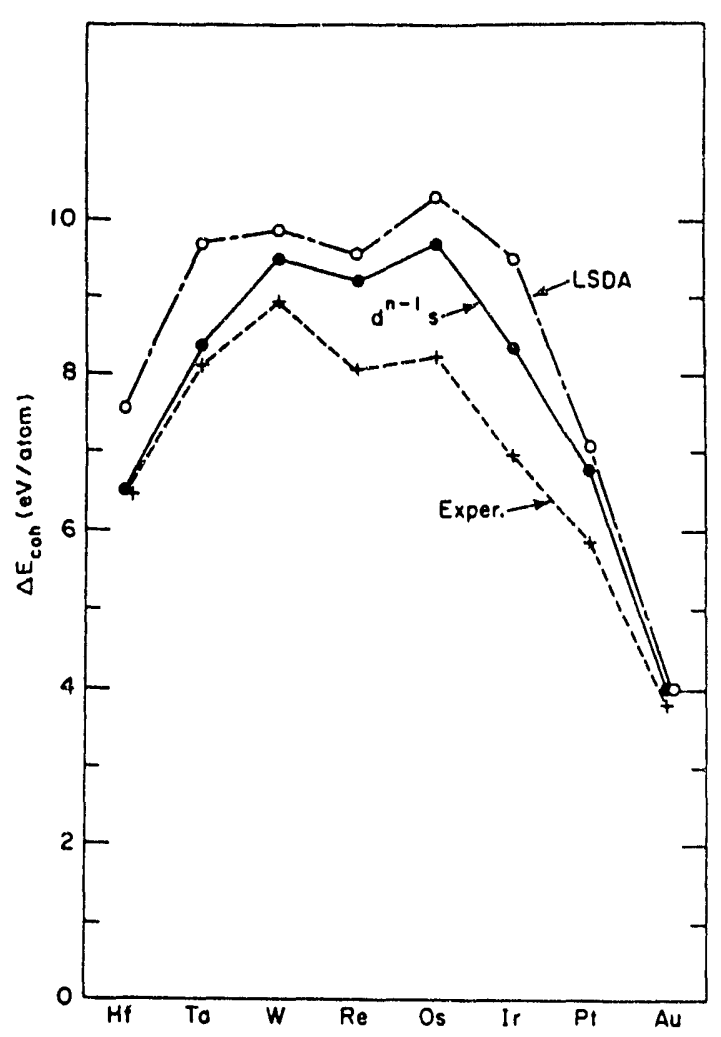

Figure 7. Calculated and experimental cohesive energies for the $5 d$ transition metals: the LSDA curve relies on the calculated LSDA ground states as the atomic reference whereas the $d^{n-1}$, employs an experimealualy based promotion energy, $\Delta p$, taking the stom from ito actual ground multiplet atate to the average over $d^{m-1}$ a levels appropriate to LSDA calculation done for the $d^{n-1}$, configuration.

\section{$s \rightarrow d$ PROMOTION ENERGIES}

Going from one atomic configuration to another involves a promotion energy, e.g.,

$$
\Delta E_{y \rightarrow d}=E\left[d^{n-1} s\right]-E\left[d^{n-2} s^{2}\right]
$$

where its experimental value enters the changed $\Delta_{p}$ and its calculated value in the $E_{\text {atom }}$ when one was calculating a cohesive energy. That is, the error in calculating 


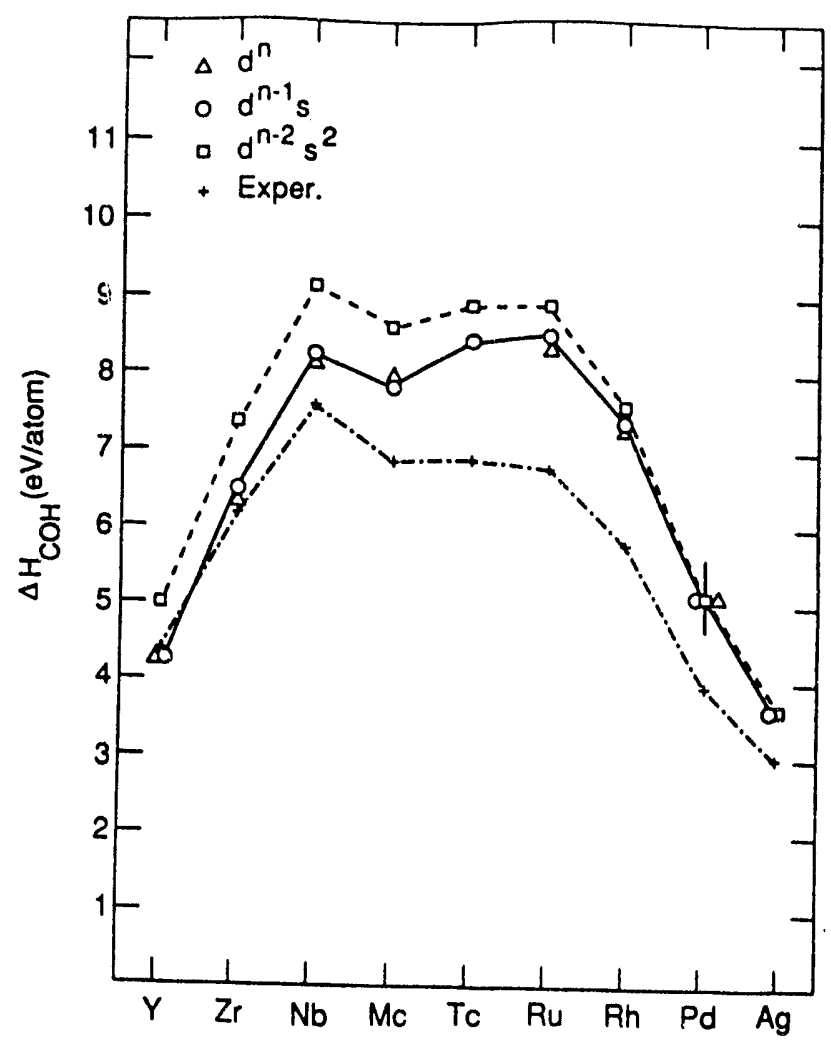

Figure 8. Calculated and experimental cohesive energies for the $4 d$ transition metals employing $d^{n-2} s^{2}, d^{m-1}$, and $d^{n}$ atomic configuraticns, in their maximum opin multiplicities, at reference states to which the promotion energy correction, $\Delta p$, has been applied.

the promotion energy

$$
\delta\left(\Delta E_{\iota \rightarrow d}\right)=\left[\Delta E_{a-d}\right]_{a b s}-\left[\Delta E_{a \rightarrow d}\right]_{c a l c}
$$

"equals" the change obtained for $\Delta H_{\text {coh. }}$. Gunnarsan and Jones studied ${ }^{20}$ LDA results for $\delta(\Delta E,-d)$. They did not have data for the $d^{n-1} s \rightarrow d^{n}$ promotions and, from what they did have, they concluded that the LDA errors arose from interactions between the valence electrons and the outer core. Such errors would imply that $\delta(\Delta E)$ should be essentially the same for the $d^{n-2} s^{2} \rightarrow d^{n-1} s$ and the $d^{n-1} s \rightarrow d^{n}$ transitions. This is not the case as can be seen in Figure 9 where $\delta(\Delta E)$ is plotted for the $3 d$ and $4 d$ row 8 . The sign of the $\delta(\Delta E)$ indicate the $d$ electrons to be too strongly bound relative to the $s$. The essential difference in the results, depending on whether or not there is an s electron common to both the initial and final state, strongly suggests that LDA's errors are associated with electron-electron interactions within the valence shell. 
- $d^{n-2} s^{2} \rightarrow d^{n-1} s$

- $d^{n-1} s \rightarrow d^{n}$

- $s^{2} \rightarrow s d \& s \rightarrow d$

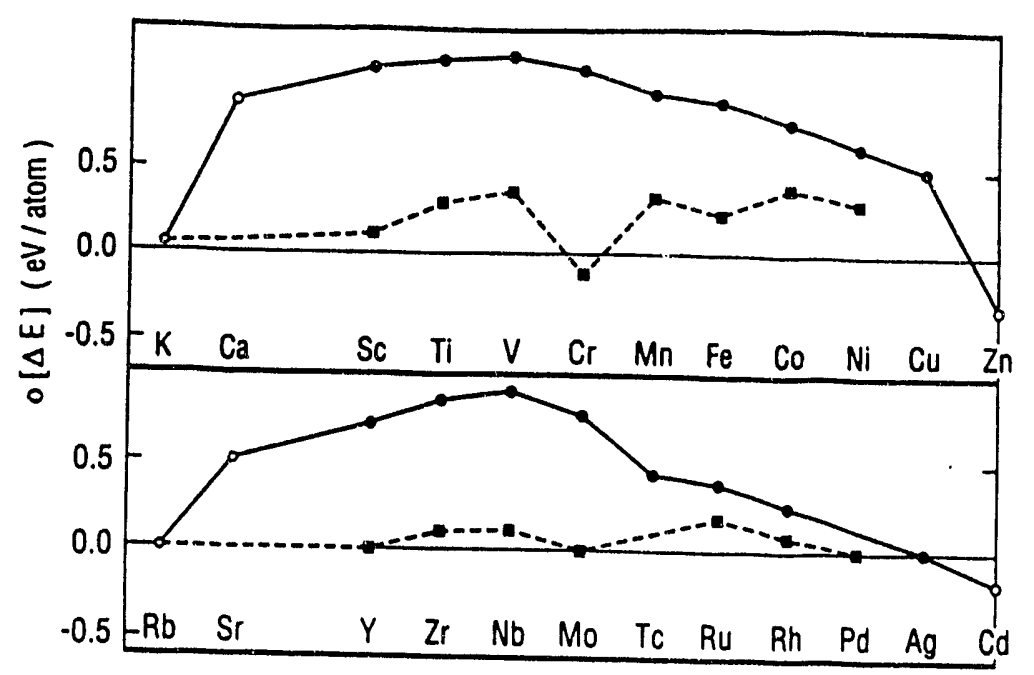

Figure 9. $\rightarrow d$ promotion energy errork, $\delta(\Delta E)$, i.e. the difference between theory and experiment group $a^{2}-$ and $4 d$ transition metal $d^{n-2} a^{2} \rightarrow d^{n-1}$ and $d^{n-1} s-d^{n}$ and odjacent main group $a^{2} \overrightarrow{-i} d s$ and $\rightarrow d$ transition for which there is reliable experimental data. (The $5 d$ $\delta(\Delta E)$ indicales sow was omitied due to a lack of $d^{m-1}, \rightarrow d^{n}$ spectral data). Note that a positive experimentally so.

\section{ORBITAL EFFECTS, HUND'S SECOND RULE}

The introduction of LSDA has allowed a treatment of the spin magnetic effects, associated with Hund's first rule within local density theory. Spin-orbit effects, hence Hund's third rule terms, are often incorporated in local density calculations (though it's commonly forgotten that spin-orbit coupling is a two-electron interaction with its own exchange ${ }^{21}$ and correlation terms which, luckily, can usually be lumped logether as an effective one-electron effect). There remains the orbital magnetic effects associated with Hund's second rule. For open $d$ and $f$ shells the energetics of orbital These terms are than, or at least as great as, the energies of spin-orbit coupling. These terms are of importance to magneto-optical effects, and they are essential to any treatment of the actinides and their compounds. The magnetism of the $3 d$ transition metal axides, in general, and the cobalt compounds, in particular, cannot be said the issue of the issue of how well does local density theory deal with describing atomic multiplet effects. The literature has concentrated $22-24$ on open $p$ shells which are a special case. Spectral data for open $f$ shells is limited and this leaves consideration of open 
d shcils, the report of which will be given elsewhere, with the sense of the conclusions given below.

Consider a $d^{m}$ configuration. We can construct an array of single determinant wave functions, $\Phi_{i}$, where orbitals of varying $m_{\ell}$ and $m$, are occupied. These may be combined

$$
\Psi_{j}=\sum_{i} b_{i j} \Phi_{i}
$$

where the $\Psi_{j}$ are eigenfunctions of $S, L, M_{L}$, and $M_{S}$. What are the consequences of taking the charge and opin density asesciated with some given $\Psi_{j}$ and evaluating ite LSDA total energy? We are already in trouble with $S c^{2+}$ with its single $3 d$ electron (or $\mathrm{Cu}^{2+} 3 d$ with its single $d$ hole) because the LSDA total energy of the ion varies by $0.2 \mathrm{eV}$ depending on the choice of the $m_{l}$ value of the electron (or hole). In other words, ISDA has states with energies varying by $0.2 \mathrm{eV}$, which in fact are degenerate cingle determinant states. Consider the multideterminant $3 d^{5}$ example represented in Figure 10. Concentrate, for now, on the ellipses which are experiment and the solid and dashed lines which are the LSDA energies for single and multideterminant $\Psi$, respectively. These lie in three narrowly defined bands depending on the $M_{S}$ of the state in question and are largely independest of $L, S$, and $M_{L}$. The ground ${ }^{6} S$ state, plotted to the i-ft shows a spread in LSDA energy of $51 / 2 \mathrm{eV}$, though according to experiment (and to Hartree-Fock theory) ihese levels are degenerate.

In addition to states which are dagent rate but have substantially different LSDA energies, there are stites which should have measurably different cnergies yet are degenerate according to LSDA. There are many such cases, e.g. in the $d^{4}$ configuration:

$$
{ }^{5} D, \quad M_{L}=1, M_{S}=0
$$

which is constructed from 6 determinants and the

$$
{ }^{3} H, \quad M_{L}=5, \quad M_{S}=0
$$

which is constructed from 2 determinants and ibe

$$
{ }^{1} I, \quad M_{L}=5, M_{S}=0
$$

which is constructed from 2 determinants, have identical charge and spin densities. Hence they have identical LSDA energies.

ISDA theory does not know up from down as far as orbital effects go because the dependence of a one-electron wave function on the sign of its $m e$ gets squared out when its density is constructed. The LSDA Hamiltonian, as we normally use it, commutes with $M_{S}$ but it does not commute with $S, L$, and $M_{L}$. This might be partially rectified by introducing a current density term to LDA. Moreover, a proper description of Hund's rule $L$ and $S$ effects involves multideterminant states - a description which lies outside band theory for solids as we nornally do it.

Slater-Condon-Racah theory describes the multiplet energies in terms of the $F^{k}$ integrals as derived in Hartree-Fock theory. The various levels of a given multiplet are degenerate as they should be though the division into Coulomb and exchange terms is not always uniquely defined. With exchange, but no correlation, the splittings between multiplet levels are overestimated. Screening the valence electron $F^{k}$ integrals by $10-20 \%$ yields predictions which are in fair acicord with experiment; the unscreened Hartree-Fock predictions plotted in Figure 10 would be in excellent accord with experiment for all but one (the ${ }^{2} D_{3}$ ) of the multiplet states with the introduction of a $14 \%$ screening of the $F^{k}$ integrals.

One may rescue the LDA predictions by imposing Hartree-Fock constraints on them. A single determinant function $\Phi_{j}$ may be expanded where the $\Psi_{i}$ are the proper 


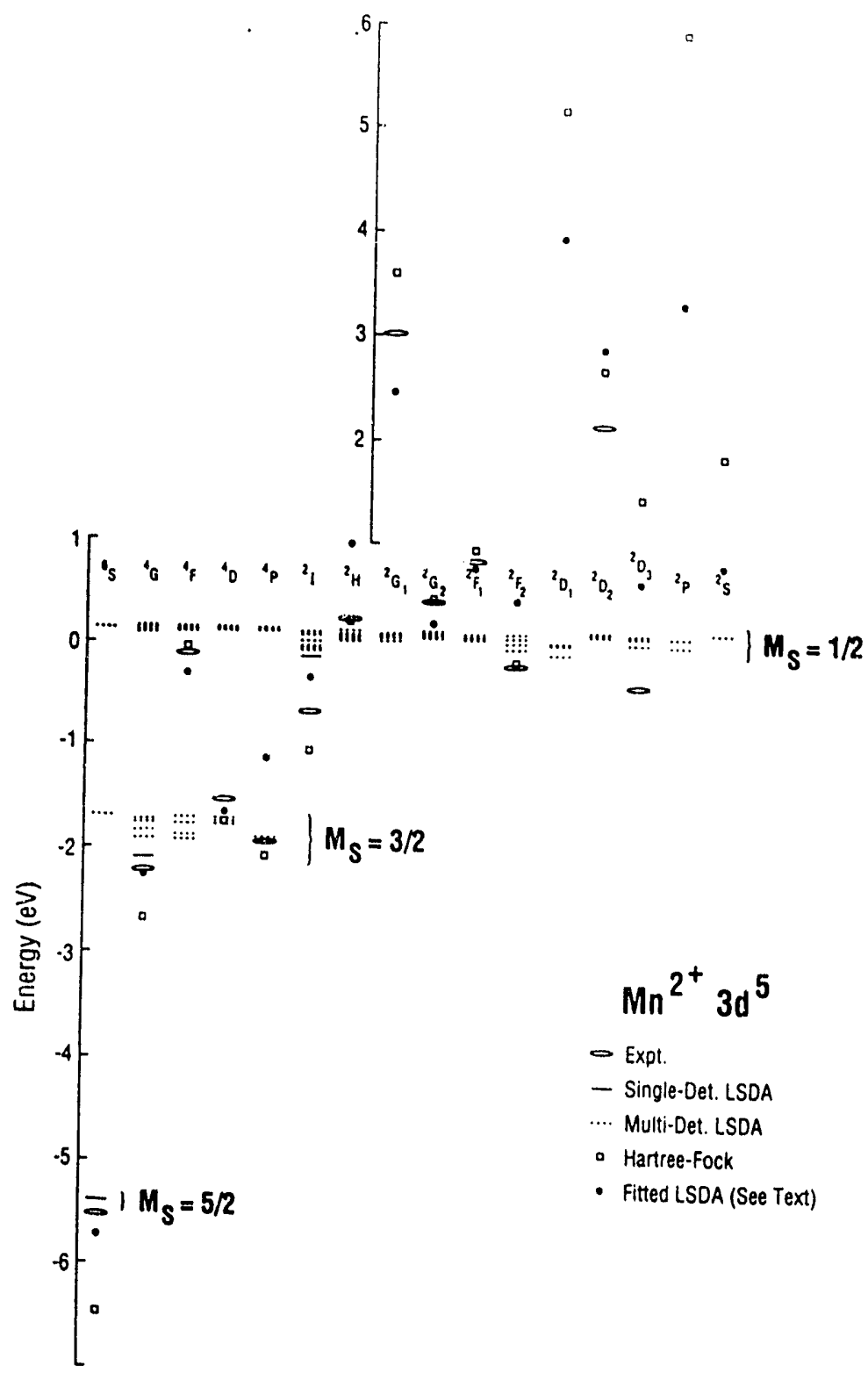

Figure 10. All the multiplet levels of $\mathrm{Mn}^{2}+3 d^{6}$ as observed experimentally and as obtained in several ways theoretically as is discuesed in text. The zero of each set of points has been taken to be the averase of the energy of that at of pointe - in the case of experiment, dat is misaing for the ${ }^{2} D,{ }^{2} P$, and ${ }^{2} S$ utates and Hartree-Fock energie for these were average (these levela have little weight in the average hence any error in using Hartree-Fock values for these ahould have little effect on the reaulting average) 


$$
\Phi_{j}=\sum \alpha_{i}, \Psi_{i}
$$

muitideterminant eigenfunctions of $L, S, M_{L}$, and $M_{S}$. Then

$$
E_{j}=\left\langle\Phi_{j}|H| \Phi_{j}\right\rangle=\sum\left|\alpha_{i j}\right|^{2} E_{i} .
$$

A set of $E_{j}$ may be calculated within LSDA and this set of equations fitted to yield $E_{i}$ subject to the constraints that those, which should be degenerate, are so. The results of such a fit appear in Figure 10. Though inferior to a screened Hartree-Fock description, the fit does trace the essential features of experiment quite well. Racah formed linear combinations of the $F^{k}$ integrals as

$$
A, B, C \text { for the } d^{n} \text { ions }
$$

where the multiplet levels of maximum spin, $S$, have energies

$$
\frac{n(n-1)}{2} A+\operatorname{coeff} \times B
$$

Similarly for the $f^{n}$ ions, Racah had terms $E^{0}, E^{\prime}, E^{2}$, and $E^{3}$, where the maxinum spin multiplet energies are

$$
\frac{n(n-1 ;}{2} E^{0}+\operatorname{coeff} \times E^{3}
$$

Here, the $A$, or $E^{0}$, term is common to all multiplets of maximum $S$ and these terms, with their $n(n-1) / 2$ prefactors include the spherical Couloumb interaction $F^{0}$. The coefficients multiplying $B$ and $E^{3}$ depend on the multiplet state in question.

Recently it has been argued that these coefficients are proportional to $L(L+1)$. One can then introduce one-electron matrix elernents proportional to

$$
m_{\ell} L E^{3}
$$

for a given orbital in an $f$ shell and to

$$
m_{\ell} L C
$$

for a given orbital in a $d$ shell.

Thus we would have a computationally simple scheme for introducing orbital magnetic effects in band calivilations. One simply adds liagonal terms of this type to muffin-tin potential terms in a band calculation. Such calculations ${ }^{25}$ have successfully reproduced observed actinide moments for actinide compounds. Unfortunately, the above argument does not bear scrutiny for open $f$ shells as may be seen in Figure 11. The separation between multiplet levels, when there is more than one, does not go as the difference in $L(L+1)$. For $f^{3}$ and $f^{4}$, the leveis are not even ordcred as a function of ascending $L$.

For open $d$ shells, it so happens that there are cases where there are pairs of levels of maximum spin multiplicity, namely

$$
{ }^{3} F \text { and }{ }^{3} P \text { for } d^{2} \text { and } d^{8}
$$

and

$$
{ }^{4} F \text { and }{ }^{4} P \text { for } d^{3} \text { and } d^{7}
$$

and their splittings, which equal $15 \mathrm{~B}$, may be written

$$
-\frac{3}{2} \Delta[L(L+1)] \cdot B
$$


Thus $d^{2}, d^{3}, d^{7}$, and $d^{8}$, if in their maximum spin nultiplicitics, are anmable to a treatment employing a

$$
-\frac{3}{2} m_{\ell} L \cdot B
$$

one-electron term in a band calculation. Norman has employed ${ }^{26}$ this for the $3 d$ oxides. There is no formal justification for employing such a scheme for open $f$ (or p) shells or for the remaining open $d$ shells.

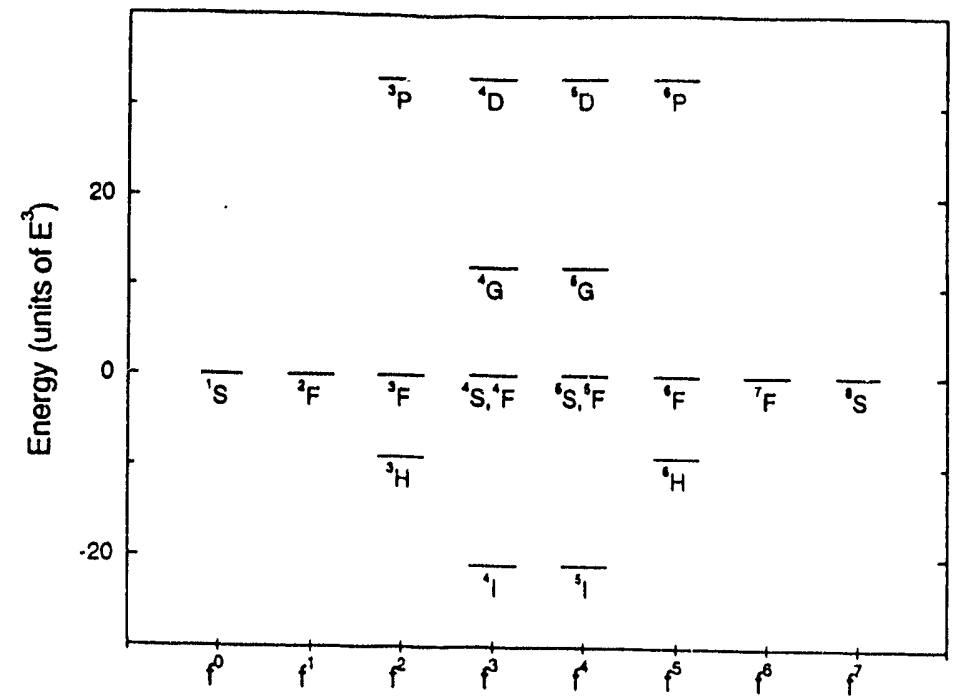

Figure 11. The multiplet splittings, in Slater-Condon-Racab theory, of $f^{n}$ multiplet levels of maximum spin multiplicity. As noted in text, this set of level splittings depends only on the Racah's $E^{3}$ term which is a specific combination of Slater's $F^{k}(f, f)$ integrals.

One way out to replace the on-site LSDA aspherical terms of a full potential band calculation by on-site Slaier-Condon-Racah aspherical terms which do know up from down, i.e. are associated with a Hamiltonian which commutes with $L, S$ $M_{L}$, and $M_{S}$. A LDA full potential calculation shares the same aspherical Coulomb terms and, having made the investment in a full potential treatment, it's not much more work including the exchange. The implementation we envisage, introduces off diagonal as well as diagonal matrix elements which are exactly derived within SlaterCondon-Racah theory.

Their remains the problem that spin and orbital magnetism is intrinsically associated with many-determinant wave functions, so as to have $S$ and $L$ good quantum numbers - something which our band structure wave function is not! 
These energy differences, e.g.

$$
E_{b c c}-E_{f c c}
$$

enter phase diagram constructs. From the Battelle-Geneva meeting ${ }^{27}$ of 1965 , ti:ere have been disagreements in sign and magnitude between band theorists and those, of the CALPHAD group, constructing phase diagrams.

Consider Figure 3, the energetics of having an $f c c I r_{0.9} M o_{0.1}$ substitutional alloy would, in first approximation, in the CALPHAD sch.sme, consist of promoting the ten percent of Mo from the bcc to the fcc structure. Similarly, the intermediate 50:50 hcp phase would, according to CALPHAD, involve promoting bcc Mo and fcc Ir to the hcp structure separately. For this to work, hcp Mo must be stabler than fcc Mo and similarly hcp Ir must lie lower than its bcc counterpart. Such ener than ignores the role that the combined $d$ band filling of the alloy plays in stabilizing this interme sate phase. At the Battelle meeting. Friedel and Mott observed that the structural energy differences appropriate to band theory are larger in magnitude than those found appropriate by CALPHAD prartitioners in constructs such as we have just sketched out. This problem remains to this day.

In Figure 12 are plotted the $b c c-f c c$ energy differences ${ }^{28}$ for the $4 d$ row. Shown are Saunders et al.'s efforts ${ }^{29}$ to push the CALPHAD-type values as close to band theory as they could - most CALPHAD workers would insist on much smaller values. While there is semiquantitative agreement for all but $R u$ and $R h$ the discrepancies are serious to standard phase diagram constructs - large energy differences causing severe depressions in the solidus-liquidus curves of the terminal phases, for example.

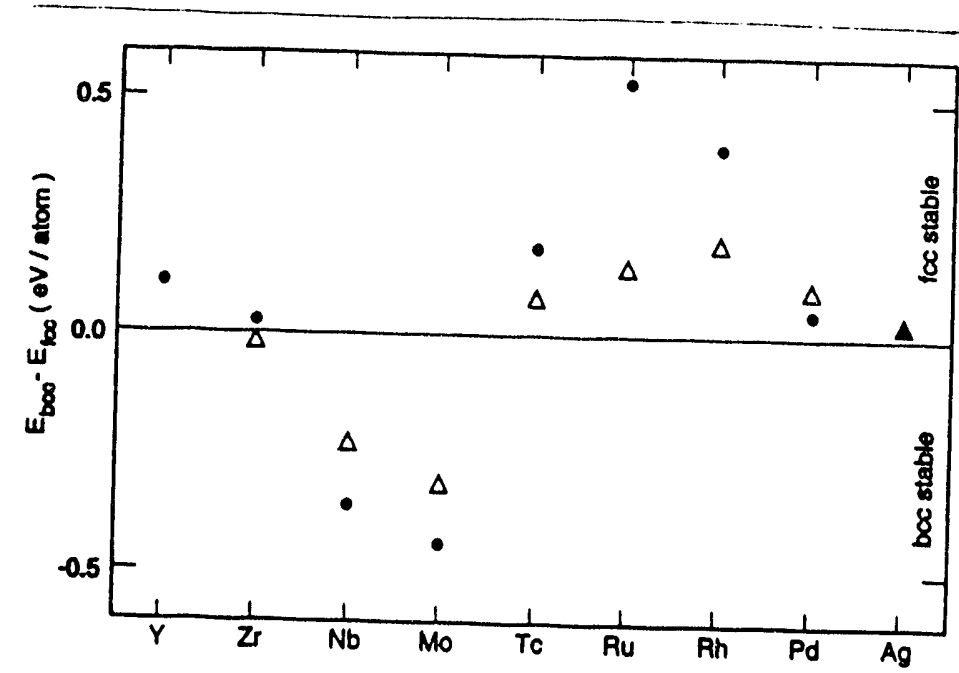

Figure 12. The bec-fec atructural energy differences for the $4 d$ transition elements. The open triangles are Saundere et a. CALPHAD-type entimates of these energy differences while the circles
are the result of full potential LASTO calculations.

The situation is even more serious for the $h c p-f c c$ energy differences of Figure 13. Note the change in scale relative to Figure 12 , the energy differences here being much 
smaller. Shown are the original estimates ${ }^{18}$ of Kaufman and Bernstein which Mott and Friedel opposed years ago along with Saunders and Miodowniks' efforts to push as close as possible to band theory. For the moment ignore the open squares. Band theory and CALPHAD disagree as to whether the bcc metals $\mathrm{Ta}, W$ (and $\mathrm{Nb}, \mathrm{Mo}$ $V$, and $C r$ ) have their excited $h(p$ or $f(c$ phases the more stable, as is important to the intermediate $h c p$ phase of Figure 3 as calculated by CALPHAD means. This sign disagreement shows no sign of going away.

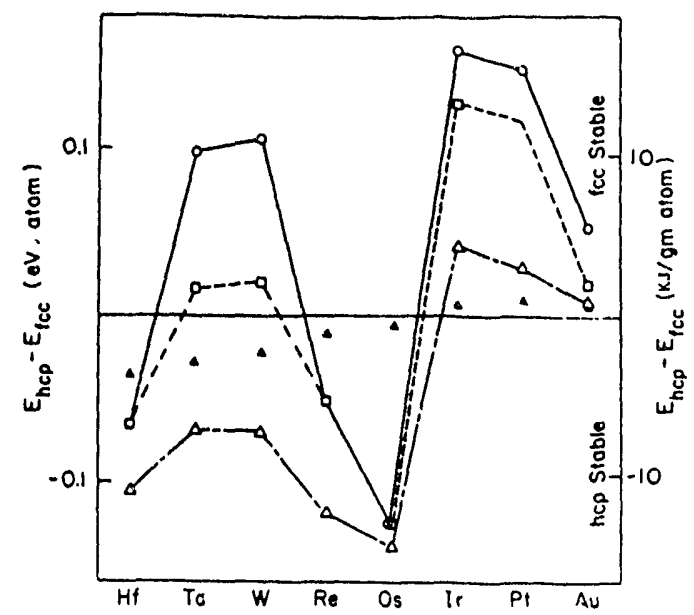

Figure 13. $f c c-h c p$ energy differences for the $5 d$ transition metals. The filt :d triangles are Kaufman and Beratein's original CALPRAD-type estimates while the open triangles : $c$ the result of Saunders et al. where they have purbed the $f c c-b c e$ and $f c c-h c p$ CALPHAD-type energy differences as close as they could to band theory. The open circles are the result of full potential fully relativiatic LASTO calculations where hcp c/a ration characteristic of transition metals were employed. See the text for a discussion of the open square LASTO results.

While less severe than years ago, the discrepancies between CALPHAD and band theory remain significant and it remains to be seen whether these represent shortcomings in the CALPHAD modelling, i.e. the thermodynamic quantities they require are not "true" quantities or in LDA based band theory to incorrectly reproduce the "true" energy differences. We do not expect that the introduction of gradient corrections in the band calculations, for example, will bring agreement on this issue.

\section{TOTAL ENERGIES AND ATOMIC POSITIONS}

The total energy of a solid depends on the lattice volume and, if the system is noncubic, on its c/a ratio. In addition, many crystal structures have atomic positions which are not set by symmetry. The $\mathrm{MoPt}_{2}$ structure, which is of concern elsewhere in the proceedings, involves an $A B B$ stacking of (110) $f$ cc lattice layers. However the lattice is, as a rule, distorted off of being strictly $f c c$, both in its $c / a$ ratio and in that $A-B$ and $B-B$ layer separations are different and these differences are of 
energetic significunce. In the Frank-Kasper and Bernal-cell phases there are, as a rule, substantial numbers of atomic positions not determined by symmetry. For example, the $\sigma(C r F e)$ phase has thirty atoms in its unit cell falling into five species of atomic sites and there are seven non-zero $x, y$, or $z$ atomic positions which may vary from one $\sigma$ phase to the next. Unfortunately, for many of the systems which have been observed crystallographically, these internal coordinates have not been dotermined or, at best, bave been ill determined in the investigation.

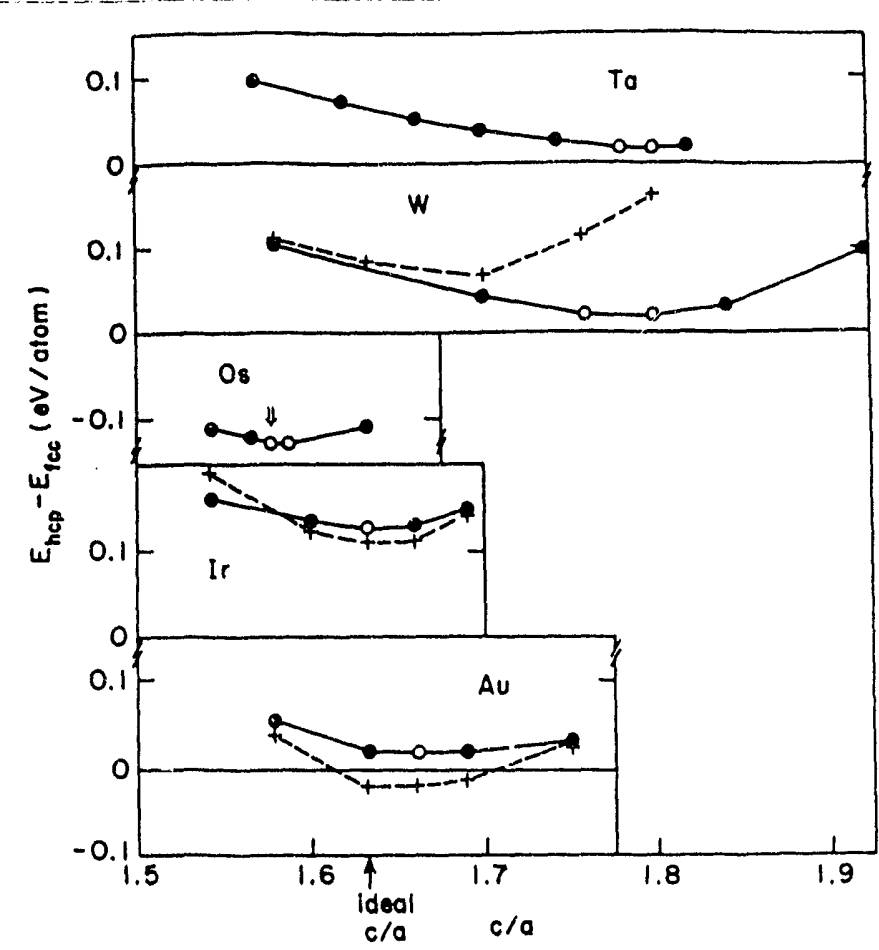

Figure 14. The hep-fce energy differences for $T a, W, O s, I r$, and $A u$ as a function of the hcp $c / a$ ratio employed in the calculations. The circles are full potential results while the + symbols indicate muffin-tin potential reaults. The open circles indicate the lowest-lying points on the full potential curves. Of the metals, only $O$. forms in the hep structure and its observed c/s ratio in indicated by the arrow in ite panel. Note, positive energy differences imply that the fac atructure is otable and negative that the hep is.

ae variation in atomic coordinates between systems which nominally form in the same crystal structure can affect the atomic coordination of the sites and this, of course, implies variations in chemical binding. In the discussion of the structural map (Figure 4) we encountered $C u A u I$ phases, most of which involved small tetragonal distortions off of an $f(c$ lattice, with the atoms having the atomic coordination characteristic of such a lattice. On the other hand, there were a few CuAuI phases, such as $I r T i$, whose c/a ratios were characteristic of a weak tetragonal distortion of 
a bcc lattice and whose atomic near neighbor distributions are characteristic of such a lattice. In the crystal phases of a number of transition metal borides, carbides, and phosphides, the metalloids are often in Bernal environments, but due to varifying internal atomic coordinates the atomic coordinations about the metalloid sites vary between systems nominally having the same crystal structure. To complicate matters further, the symmetries at these sites are often low implying that it is harzardous to do a band calculation which does not employ a full potential.

In Figure 14 is plotted the hcp- $f$ cc structural energy differences calculated for several $5 d$ metals, as a function of the hcp $c / a$ ratio. Of these, only $O s$ forms in the $h c p$ structure and the minimum in the energy as a function of $c / a$ is in good accord with experiment and in accord with the $\mathrm{c} / \mathrm{a}$ ratios observed for the other transition metals which form in the structure. It was such $\mathrm{c} / \mathrm{a}$ values which were used for the open circle results of Figure 13. The sergy minims for the other metals, not normally forming hcp structures, fall well away from the $c / a$ characteristic of $O_{s}$ and its hcp transition metal counterparts. The $c / a$ minima obtained for $T a$ and $W$ are for $c / a$ well above the ideal $c / a$ for $h c p$ systems: such large $c / a$ are encountered in $Z n$ and $C d$. The open squares plotted in Figure 13 are the result of minimizing the hcp energies as a function of $c / a$. The hcp-fcc energy differences for bcc $T a$ and $W$ are much closer to zero but their ign is still inconsistent with CALPHAD expectations. Muffin-tin potentials results, the crosses, are shown for several of the metals in Firure 14. We see that a muffin-tin treatment erroneously indicates $h c p$ Au to be more stable than $f c c$. Finally, we should note that variations of as much as $0.1 \mathrm{eV} /$ atom attended the variations of $c / a$ and this is of a scale to be of concerm when making total energy predictions. .

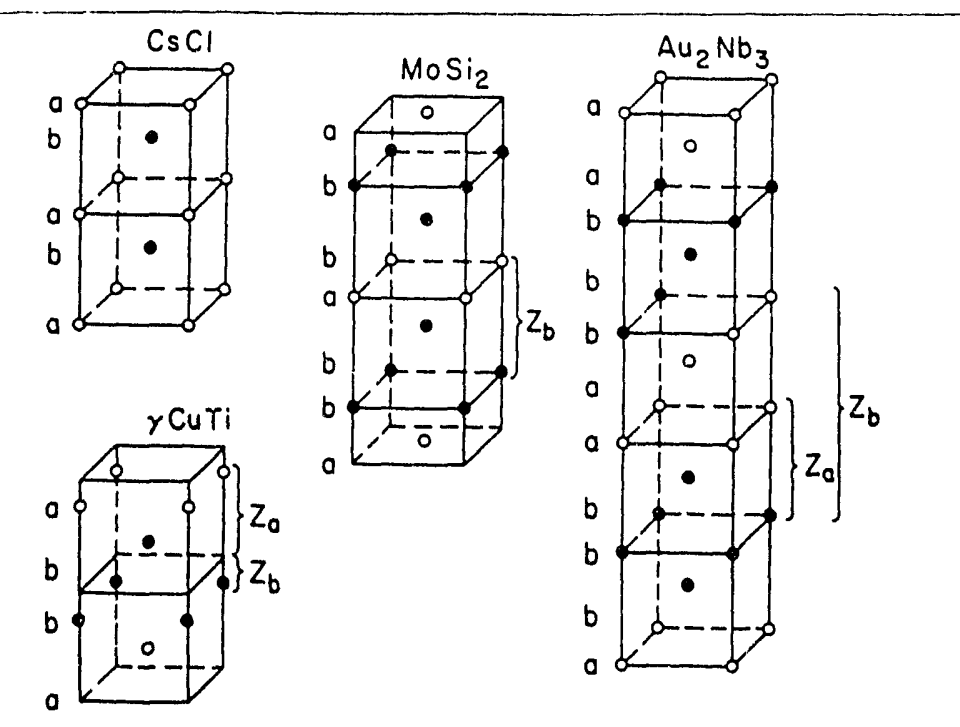

Figure 15. Some layered bec-like crystal strucutres encountered as ordered Au- and Pt- $5 d$ metal phases. Single unit celle are shown, except for the $\mathrm{CsCl}$ structure where two unit cell are drawn. Open circles indicate one species of atonu and filled the other. 
A large number of ordered alloy structures are associated with the filling of $b c c$ or $f c c$ lattices which then distort because of the atomic occupancies. Several sy'stems which involve the ordering of $(100)$ layers on a bcc lattice appear in Figure 15. Except for the $\mathrm{CsCl}$ structure, the layering causes the $\mathrm{c} / \mathrm{a}$ to deviate from that strictly appropriate to a bcc lattice. For example, $A u H f_{2}$ and $A u_{2} H f$ form in the $\mathrm{MoSi}_{2}$ structure with $\mathrm{c} / \mathrm{a}$ of 3.59 and 2.45 respectively whereas the ideal $\mathrm{c} / \mathrm{a}$ is 3 . Those deviations can be understood in terms of the fact that $H f$ is larger than $A u$ thus affecting the in plane versus interplane spacing. The $Z$ 's of the figure define measures of the variation in interplanar spacings. Binding energies, calculated as a function of the $Z$ 's, appear in Figure 16 for $A u H f_{2}, A u_{2} H f, A u H f(C u T i)$, and $A u_{2} T_{a_{3}}\left(A u_{2} N b_{3}\right)$. The calculations were done at the crystallographically observed volumes and $c / a$ ratios (which are 1.82 and 4.51 versus ideal values of 2 and 5 for the latter two compounds). These are muffin-tin potential results and when they were done $^{30}$ there were no experimentally determined $\Delta H$. Calorimetric values became available ${ }^{31}$ last year, as indicated by the horizontal arrows, and these are in satisfying accord with the minima obtained for the heats. Crystallographically based $Z$ values were quoted only for $A u_{2} H f$ and this value, of 0.34 , has an uncertainty of at least \pm 0.01 . The horizontal brackets in the figure indicate the range in $Z$ values quoted for other systems having the same crystal structures. The minima in the curves are thus consistent with what little is known experimentally. Only in the case of $\mathrm{Au}_{2} \mathrm{Hf}$ do this minima conform with a regular spacing between atomic layers and large energies are associated with the deviation from regular spacings $\left(Z_{c}-Z_{b}=1 / 4\right.$ and $Z_{a}+Z_{b}=1 / 2$ for $\delta C u T i, Z_{b}=1 / 3$ for $M o S i_{2}$, and $Z_{a}-Z_{b}=1 / 5$ for $\left.A u_{2} N b_{3}\right)$ for the other phases. For $A u H f_{2}$ this energy exceeds $1 / 3 \mathrm{eV} /$ atom. The strong energy dependence seen here can be understood since changing interplanar spacings corresponds to the energetics associated with optical phonons.

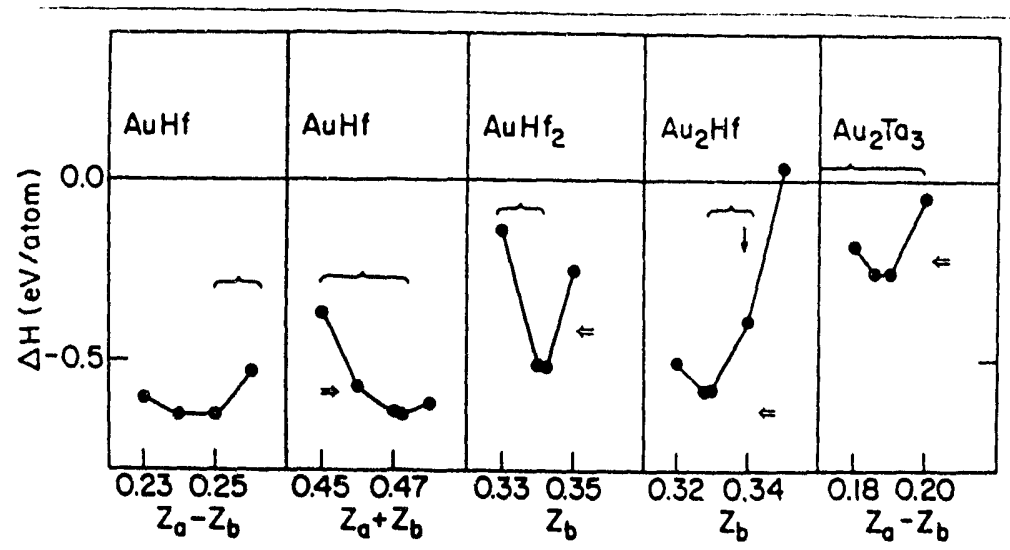

Figure 16. Heate of formation of $\mathrm{AuHf}\left(\delta-\mathrm{CuTi}\right.$ structure), $\mathrm{AuHf}$ and $\mathrm{Au} \mathrm{u}_{2} \mathrm{Hf}\left(\mathrm{MOSi}_{2}\right.$ structure), and $\mathrm{Au}_{2} \mathrm{Ta}_{3}$ calculated as a function of the $Z$ coordinates defined in Figure 15 . The horizontal arrows indicate the experimentally meanured bents. The $Z$ 's have not been determined experimentally, sxcept for $A u_{g} H f$ whow $Z_{b}$ is indicated by the vertical arrow and whose uncertainty is $\sim 0.01$. The brackets indicate ranges of pomible $Z$ values as inierred for other systems forming in the same structures. 
As another example of the energetics associated with a distortion off of a wellpacked lattice, consider the $P t_{8} T i$ structure sketched in Figure 17. This is one of the phases whose binding energy is featured in Figure 1. The structure is an $f c c$ lattice if $a / c=3 / \sqrt{2}$ and if $x_{1}=x_{2}=1 / 3$. Pt $t_{8} T$ has a slightly larger a/c ratio than this and $x_{1}=0.327$, due to $T i$ being smaller than $P t$, with $x_{2}=0.333$. The heat represented in Figure 1 was the result of a full potential calculation employing these coordinates. The prototype system having this structure is $V_{1} Z n_{5}$ with $x_{1}=0.348$ and $x_{3}=0.328$. The $P t_{8} T i$ energy was calculated with these $x$ 's as well, resulting in an $0.02 \mathrm{eV} /$ atom loss in binding. While the $x$ dependence was not explored more thoroughly, it would appear that the energy dependence of lattice deviations is small here, i.e. hundredths of an $\mathrm{eV} /$ atom in comparison with the tenths of an $\mathrm{eV} /$ atom encountered in the layered structures.

The issue of displacement energies is of importance to the modelling of ordered and disordered alloys as discussed by Stocks and by Zunger elsewhere in these proceedings. Such displacement energies along with the question of the role of lattice positions in the energetics of ill-packed Frank-Kasper and Bernal environment phases, complicates any investigation of the metastability or stability of ordered phases involving such structures. The examples above suggest that the effect on binding energies will sometimes be minor and sometimes severe. With more experience, we will likely learn where it is most important to take care.
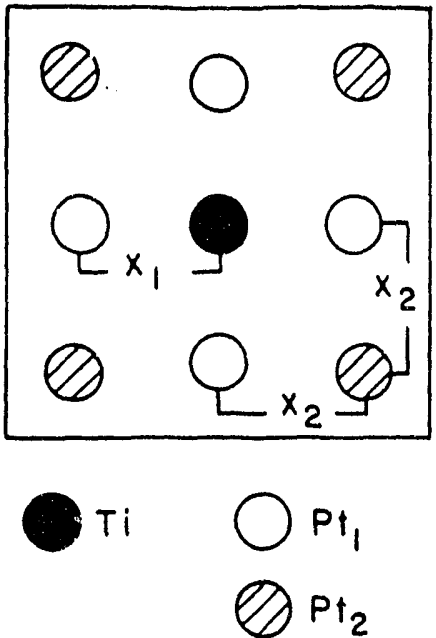

Figure 17. One layer of the body-centered Pt.Ti structure (in the other layer, the Ti's lie on the corners of the square). Depending on the $a / c$ value, the structure may correspond to an $f c c$ or bec lattice or neithes. The $f c c$ occurs when $a / c=\sqrt{3 / 2}=2.1213$ and when $x_{1}=x_{2}=1 / 3$.

\section{CHARGE TRANSFER}

It has been common to attempt to rationalize the bonding and other properties of solids, such as their elastic moduli, in terms of the valence charge, namely in terms 
of charge transfer and of the directionai character of the bonding charge. The latter is most usefully done for covalently bonded systems. Charge transfer is often thought

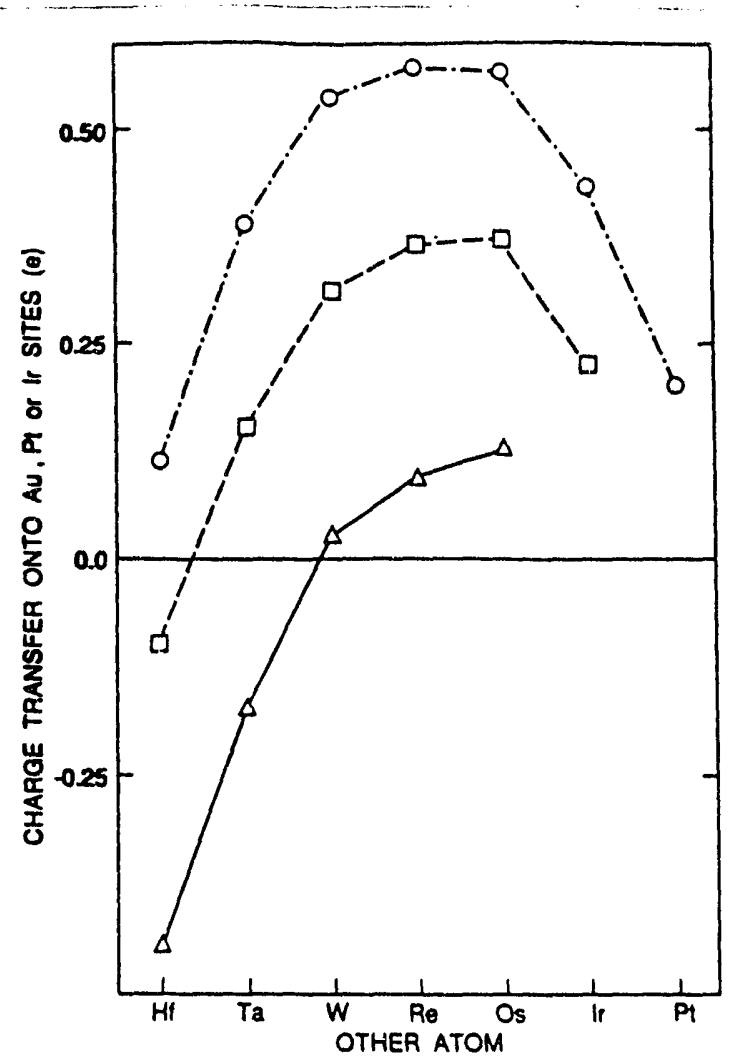

Figure 18. Charge trunsfer on of of Ir (triangle), $P t$ iequare), and Au (circle) calculated for compounde of these atoms with other $5 d$ elements having the $\mathrm{CsCl}$ structure. The charge at a site is obtained by integration over the charge in the Wigner-Seits uphere and the tranefer obtained by comparing the charge at the site in the compound with that of the elemental metal. A positive sign. indicates eloctron fow cato $\& A u, P t$, or Ir vite. Calculations were done for the elemental metals and the compounds at their obeerved lettice volumes: when the $\mathrm{C} \& \mathrm{Cl}$ etructure does not occur but another $1: 1$ eystem doen, ite volume was ueed. For cases where there is no reported eryatallographic date af 1:1, then the pattarn of how the molecular volume deviates from the oum of the elemental volumes was uned at the bacis for interpolating a compounda's volume. Given that volume, the elemental Wignemseits ophere volumes of the two alloy constituente were multiplied by a common scale factor wo that the num of their volumes equals the molecular volume.

of in terms of electronegativity trends and it is generally accepted that electronegativity increasea, i.e. the propensity to attract valence electron charge increasea, as the transition metals are traversed from left to right. Disagreement occurs with the noble metals where the Pauling electronegativity scale has $A u$ the most electronegative of the metals while the metallurgist's work function scales have the noble metals less 
electronegative than the transition metals to their immediate left. It would seem easy to measure this. Consider taking the charge distribution resulting from a band calculation or from the density obtained by $x$-ray diffraction and to integrate over an atomic cell for the charge centere $\downarrow$ on a site. Problems arise in chrosing what volume to attribute to what site in a compound and whether all the charge found at that site really "beiongs" to the atom centered there.

Consider Figure 18 where the charge transfer has been calculated ${ }^{32}$ for $I r, P t$, and $\mathrm{Au}$ compounds taken in the $\mathrm{CsCl}$ structure, a structure where the eight nearest neighbors are unlike neighbors. Except for the $H f$ compounds these systems pretty well obey Vegard's law, that is the volume of the molecular unit in the compound equals the sum of the elemental volumes. In such a case, the sites are given their elemental volumes and when the molecular volume is less than the sum, the volumes of the two sites are each scaled accordingly. The gold curve lies highest consistent with $A u$ being more electronegative than $P t$ or $I r$ however all three curves bend down and if the results had been extended to $L u, L u$, or a rare-earth between, $A u, P t$, and Ir would all be seen losing charge to this electropositive element. This is contrary to any view of charge transfer behavior and is not an idiosyncrasy of this particular set of calculations. Others have done calculations ${ }^{33}$ for the $N d-F c$ hard magnets which show charge transfer from $F e$ onto the electropostive $N d$. Sense will be made of Figure 18 shortly.

\section{GOLD ALLOYS AND \& - d TRANSFER}

For several thousand years, goldsmiths have known that alloying $A g$ into $A u$ causes a rapid whitening in color. This is associated with a drop in $A u$ d bands with respect to the Fertin level. More recently, photoemission has shown that the $A u 5 d$ bands and, more slowly, the core levels drop when $A u$ is alloyed with a number of other metals. (The more rapid shift of the $d$ bands is due to their active role in the bonding.) A drop in levels implies a deepening potential and this, in turn, indicates a depletion in bonding charge at the $A u$ site. This is contrary to the notions of Pauling and others that $A u$ is the most electronegative of the metals.

Gold has a nucleus ammenable to Mössbauer effect measurements and the resulting isomer shifts show the $A u$ contact densities increasing in the same alloys. This indicates s-electron transfer onto $A u$ opposite to that suggested by the energy level shifts. Invoking separate $s$ and $d$ valence electron transfer terms, and employing ${ }^{34}$ both calculations and experiment, it was concluded that Au alloying involves: $s$ transfer onto and $d$ off of gold sites, where there is a resulting small net transfer of charge onto $A u$. Being more compact, the $d$ levels make larger contributions to the Coulomb potential and, hence, their Coulomb contributions are more important to the electron energy level shifts.

The $A u d$ bands are completely occupied, lying 2, and more, $\mathrm{eV}$ below the Fermi level. Nevertheless, $A u d$ bonding is important chemically. This is due to hybridization of unoccupied wave function character from the other alloy constituent into the occupied $d$ bands. This depletes the occupied $A u d$ electron count. The band staye flled, but the $d$-count in it decreases. Whatever the $d$ electrons do, it is plausible that the leas local non-d valence charge will, among other things, act to screen any $d$ count change. The simple picture of $s-d$, or $d$-non-d charge compensation at $A u$ sites appears to be sustained by experiment, though the situation is more complicated, as will ahortly be seen.

Going to $P t$ and $I r$, there is also a tendancy to fill their almost full $d$ bands. This accompanies an on-site $d$ electron depletion due to hybridization into the already 
occupied $d$ bands and the result is to be seen in Figure 19. While the $A u d$ bands have lost on-site d-count due to hybridization, the combined effect of band filling and hybridization has a near zero effect on Pt's and Ir's $d$ electron counts. The $\Delta n_{d}$ of the atoms alloyed with $I r, P t$, and $A u$ are perhaps surprising, for these atoms show $d$-count losses. What is clear is that the $d$ electron count of the two atom types in a compound is not preserved, implying that the energetics of this transition metaltransition metal alloying cannot be completely understood in terms of $d$ band bonding alone.

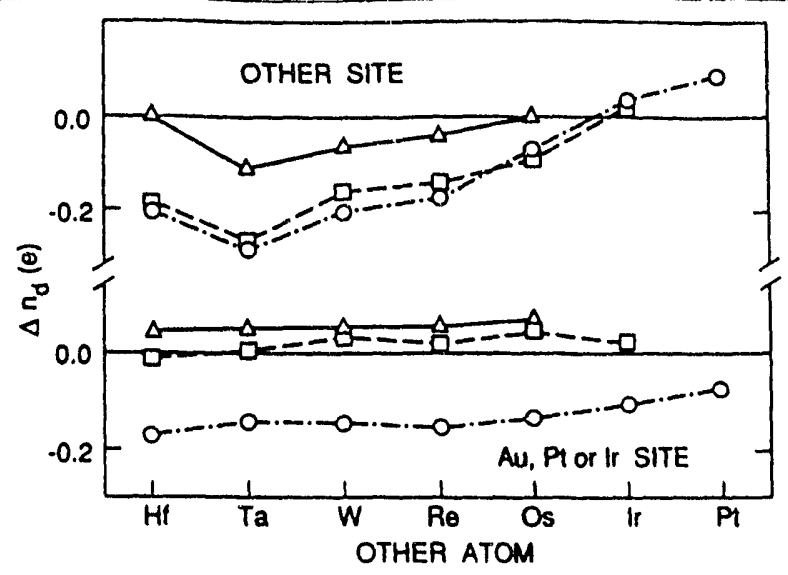

Figure 19. The calculated d electron count thange, within the Wigner-Seitz spheres at the Au (circle), Pt (equare), and Ir (triangle) sites and at the other wiles to which they are alloyed for the $\mathrm{CaCl}$ structures represented in Figure 18.

\section{CHARGE TAILING}

The wave function components within the atomic spheres are carried up to quite high $\ell$ in an augmented basis set calculation; $\ell \leq 8$ was used in the LASTO calculations reported here. The high $\ell$ terms are real components of the charge density and significant charge resides in them as can be seen in Figure 20. Similar results have boen obtained ${ }^{30}$ in LAPW calculations for the same elemental metals. Now we do not expect $f, g, h, i$ - bands in these $5 d$ metals. Let us assume that this charge, $n_{Z^{3}}$, is associated with the tailing of charge intrinsic to near-neighboring sites.

Then in an $A B$ alloy in the $\mathrm{CsCl}$ structure, where the eight nearest neighbors are unlike neighbors, one might expect

$$
n_{l^{3}}^{B}(\text { alloy })=\frac{V^{B}}{V^{A}} n_{\ell^{3}}^{A}(\text { elemental })
$$

i.e. the tail density of neighbors $A$ scaled by ratio of the volume sampled by $B$ in the compound, relative that sampled by $A$ in its elemental state. The result of this assumption is compared in Figure 21, with the actual $n_{\ell}^{B} \geq 3$ obtained in calculation for the compounds. The agreement is remarkable. It would appear that the $n_{\ell_{2}}$ charge terns arise from "tailing ${ }^{n}$ from an atom's neighbors. The change in these 
contributions upon alloying is substantial as is seen for the Pt compounds in Figure 22. These terms are of the order of the $d$ electron count changes of Figure 19

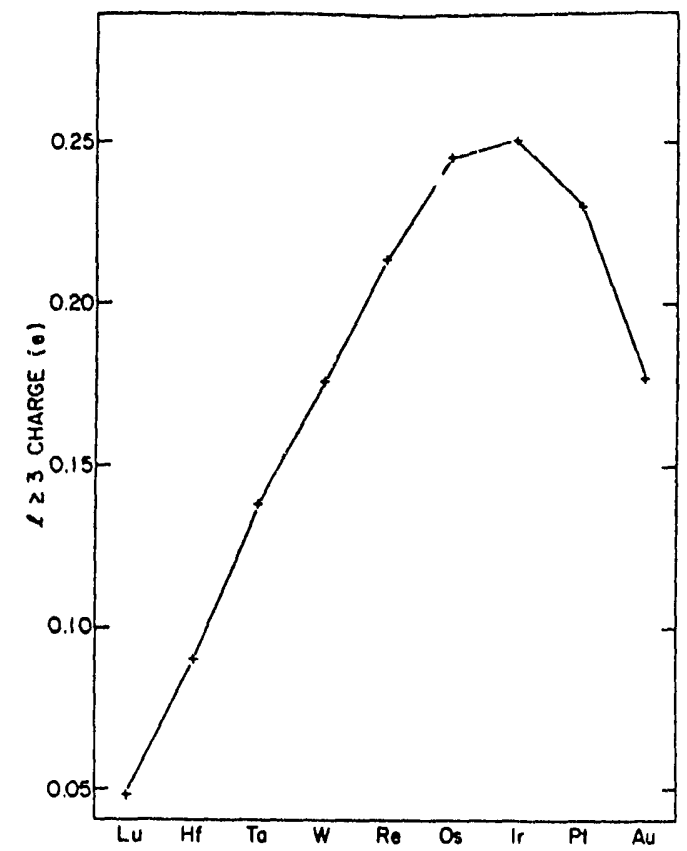

Figure 20. The tolal charge, associated with wave function components of $\ell \geq 3$, residing in the Wigner-Seitz spheres of the elemental $5 d$ metals as obtained with LASTO band calculations.

What of $s, p$, and d-like charge tailing terms? In expanding a Bloch sum at some given atomic site, within the augmented basis set scheme, it is possible $\mathrm{e}^{32}$ to separate the wave function into on-site and off-site contributions, with the tailing terms correctly orthogalized to the atomic core. Squaring and summing the wave functions yields on-site, tailing and overlap terms. Quantitative details depend upon the choice of the basis set in the interstitial region, but this does not affect the overall conclusion. More serious, is the question, well known to quantum chemists, namely how to apportion the overlap charge density. Quantum chemists in their orbital population andysea often employ a Mulliken scheme where the overlap contribution is divicied equally. This seems implausible here for the $d$ term of transition metals where there can be on-site $d$ counts approaching 10 , while the tail terms are much less. We will employ a "modified Mulliken" scheme where the overlap charge is apportioned proportional to the on-site and tailing terms. The result of this appears in Figure 23 where tailing effects have been subtracted from the overall charge transfer terms of Figure 18. Having made the subtraction, the results of Figure 23 provide a measure of the "chemical" effects associated with band filling (or emptying), hybridization and 
the charge screening of these and of the tailing terms. Consider the top panel first. All other alloy components lose charge to $P t$ and $I r$, losing more to $P t$ than to $I r$.

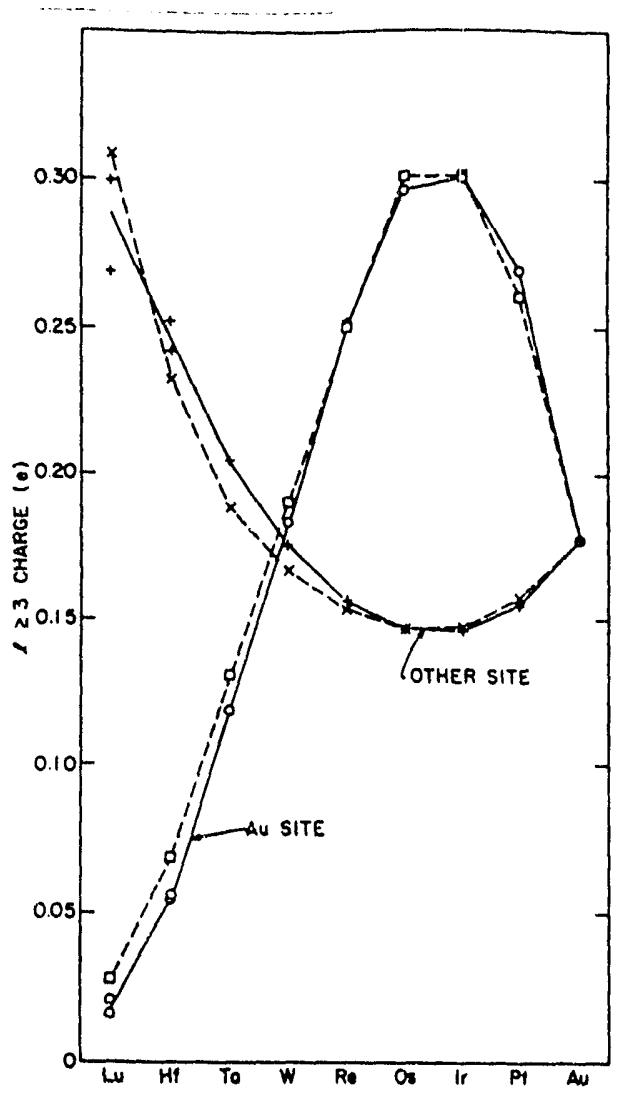

Figure 21. The charge in $\ell \geq 3$ components, within $A u^{\prime}$, and the ober element'n Wigmer-Seits spheres as obtained for the Au-based compounde, in the $\mathrm{CsCl}$ atructure, represented in Figure 18. The solid lines are the results obtained directly from band calculations for the compounda while the dasbed curves are eatimatea based on realing the elerrental $\ell \geq 3$ charge components of Figure 20, as diecused in text.

In addition, $O s, I_{r}$, and $P t$ have all gained charge when alloyed with $A u$. In the lower panel the $P_{t}$ curve now lies highest and except for the bend over for $\operatorname{Ir} H f$ and IrTa, the $A u$ curve lies lowest with $A u$ losing charge to $R e$ as well as to $O s, I r$, and $P t$. These charge transfer trends make sense when taken in the light of work function based electronegativity scales where $A u^{\prime}$ 's electronegativity is found to be less than that of Re, Os, Ir, and Pt. The results are incompatible with the Pauling scale which has $A u$ the most electronegative metal. Of course it is the total charge at a site, i.e. these terms plus the charge tailing, which is sampled by an experiment. 
$A u L i$ and $A u C s$ form in the $C s C l$ structure, though with violent deviations from Vegard's law. The molecular volume of $A u C s$ is less than that of $C s$ in $C s$ metal and similarly the molecular volume of $A u L i$ is less than the atomic volume of $L_{i}$ metal.

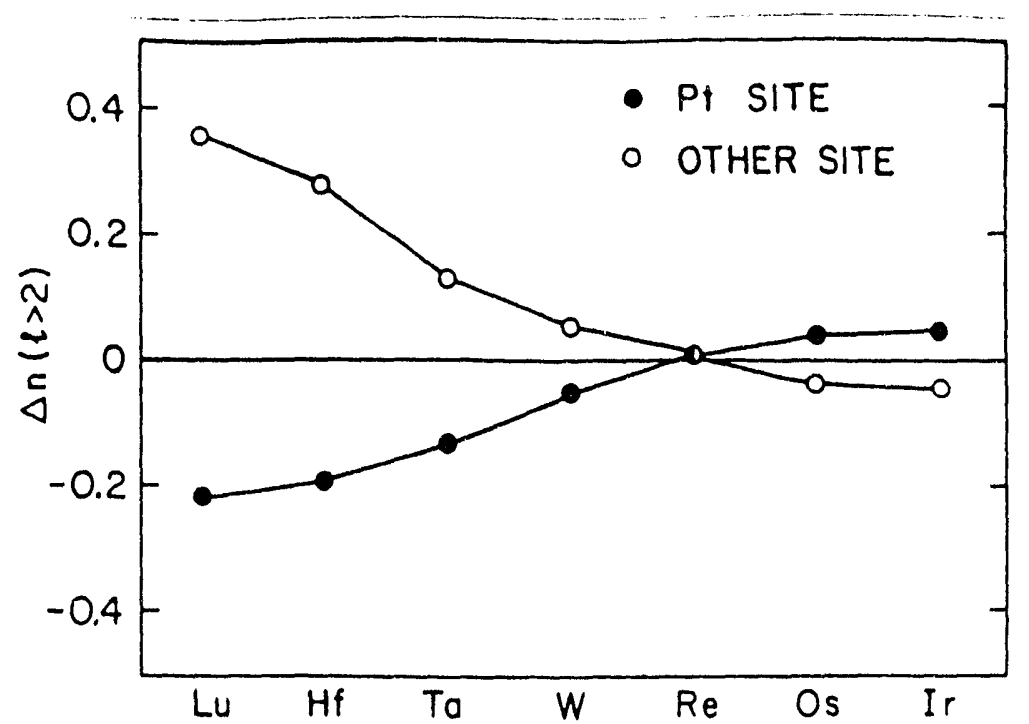

Figure 22. The change in charge in the high $\ell(l>3)$ components on taking $P t$ (and the otber $5 d$ metal) from its elemental metallic form to the $P_{t-5 d}$ systems in the $C_{s} C l$ structure.

When inspecting these systems' charge transfer, we will attribute $A u$ metal's volume to the $A u$ site in the compound and the remaining molecular volume to the alkali. This should bias any estimate of charge behavior in the direction of having placed too much of the charge on the $A u$. The total charges broken up into their $\ell$ components are plotted for these compounds and for the elemental metals in the top panel of Figure 24 while the tail charge, alone, appears in the bottom panel. A striking feature of the lower panel is the extent to which the tail charge of the alkali in the compound resembles that for $A u$ in $A u$ metal while that for $A u$ in the compound resembles that of the elemental alkali metal. In addition, the total tailing charge, summing over $\ell$, is substantial at the allali sites in the compounds. As for the total charge, in $A u C s$ the $A u$ atoms are rather well separated and reside in something approximating a $d^{10} \mathrm{~g}$ configuration while in $A u L i$ the $A u$ are closer together causing significant $A u-A u$ bonding. This resulte in a profile in $\ell$ components much like that of elemental $A u$. The large $d$ component soen for $C s$ in $A u C s$ is associated with $C s$ 's $d$ bands which lie in the states close above the band gap (the system is a semiconductor). These states have hybridized with the occupied bands, causing the enhanced $d$ electron count.

In terms of valence charge at the site, the alkalis in their compounds are negatively charged. But, in terms of valence orbitals centered on a site, the alkalis (and $N d$ in $N d F e$ or $T i$ in $P t T i$ ) are positively charged.

Consider an alloali atom adsorbed on a transition metal surface. Where here, in the solid, the alloali had eight nearest neighbors, at an adsorption site it will, typically, 
have 4 or $\dot{6}$. The surface alkalj site can also be expected to be negatively charged - not hecause of charge transfer or hybridization - but simply because of charge taiiing into the site. Arguments abound concerning the ionic versus covalent character of the bonding associated with adsorbed alkali atoms. Important to the numerics of where the charge is, is the charge tailing onto the alkali site. This will affect such argumentation.

Charge tailing would appear to be one important source of "charge transfer." It can cause positive ions to have net negative charge at their sites and vice versa. It combines with "truen chemical charge transfer in any experimental measure of where the charge is. The earlies observations concerning $A u$ alloys involve this combined charge.

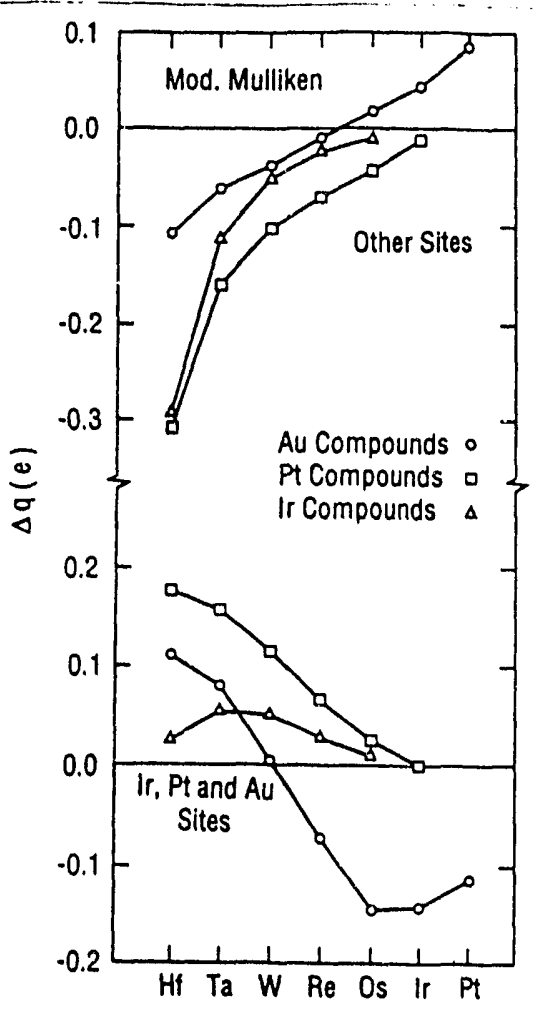

Figure 23. Charge tranafer, for the ayatema repreacnted in Figure 18, after charge tailing has been subtracted off employing the modified Mulliken scherne. Nole, positive value indicatea a net increase in electron count at the site.

\section{THE HEATS OF FORMATION OF THE ORDERED COMPOUNDS}

The calculated heats of formation of the $I r, P_{t}$, and $A u$ compounds, of Figures 18 and 23, appear in Figure 25. Significant binding is seen for the $H f$ compounds and 
nonbinding once $W$ is reached. The $C s C l$ structure is a good representative for the left hand compounds but not for those of the right, but it does indicate certain essential
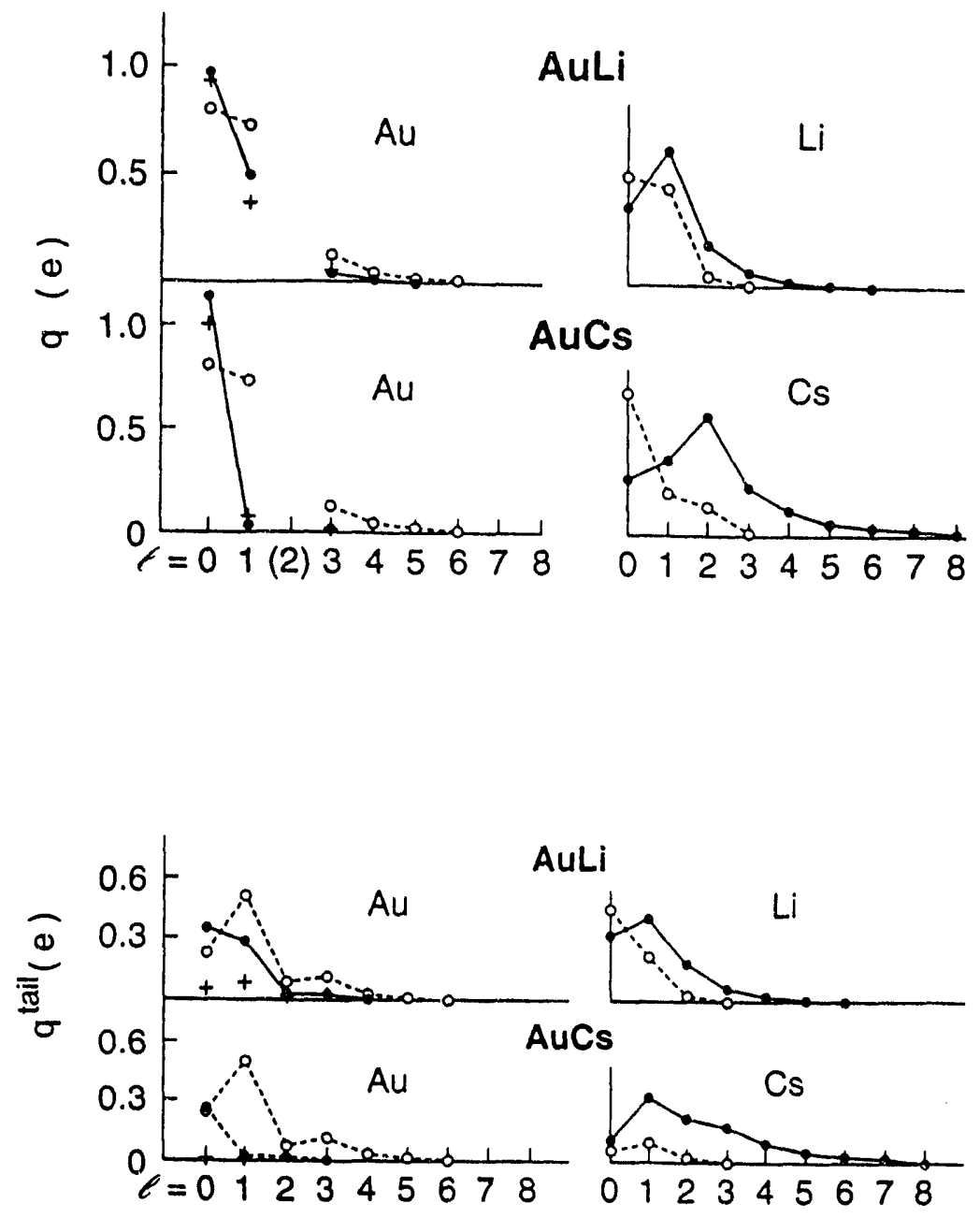

Figure 24. The $\ell$ components of charge for AuLi and AuCs calculated at their obecrved lattice constante in the $\mathrm{CaCl}$ atructure. The upper panel shows the lotal charge of some $\ell$ value within the Wigner-Seitz sphere (Au's $5 d$ charge is not plotted since it falls well outside the figure). The lower panel show that component of the charge which is associated with tailing having employed the modified Mulliken scheme. The filled circlea are the results for the compounds, the open circles results, for comparion, for the elemental metals and the + 's are results for simple cubic $A u$ at the Au-Au spacinga appropriate to the compounds (i.e. the Au lattices in the compounds, baving emptied out the albali'a). In the compound, Au was assigned its elemental Wigner-Scitz volume and the remaining, much contracted, molecular volume was assigned to the alkali. 
features of the binding trends. Strong binding is to be found to the left and weak binding is to be found to the right hand of the curves. The weak binding is manifested by the full substitutional solubility in the $\operatorname{Ir} P t$ and $A u P t$ systems with the latter ordering at low temperatures. That the $A u$ alloy curve lies highest is consistent with phase diagram behavior where $A u-W, A u-R e$, and $A u-O s$ are immiscible while their $P t$ and $I r$ counterparts at least show substantial ranges of terminal solubility. The strong compound formation, seen to the left, is readily understood in terms of $d$ band filling considerations as is seen for the $P t$ alloy densities of states appearing in Figure 26. In $L u P t, H f P t$, and $T a P t$ we have double peaked densities of states involving low-lying bonding $P t$-like states and high-lying nonbonding levels which are primarily associated with the other constitient.

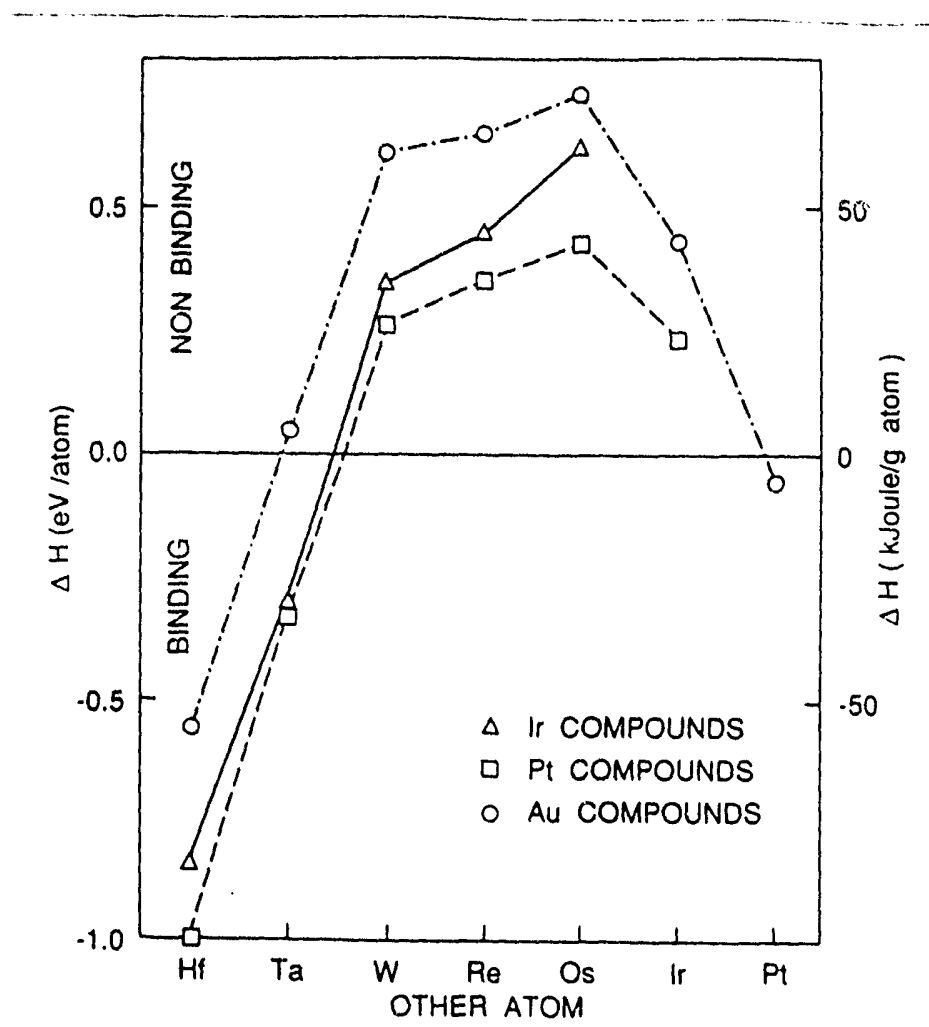

Figure 25. Calculated heats of formation of 50:50 compounds in the CsCl structure of $A u, P t$, and Ir allowed with the $5 d$ elements.

IrPt on the other hand, shows bands while double peaked, which are strongly mixed in Ir-Pt character. In $L u P t$ and $H f P_{t}$ the bonding levels are filled and the Fermi level falls at the lower edge of the nonbonding states and a strong heat of formation ensues. By PtTa more nonbonding states are filled with a consequent loss in bonding energy. By $W$ or Re everything has been pretty well lost. Coupled with the fact 
that the competing elemental $W$ and Re phases are themselves strongly bound due to having near half-filled bands, the formation of $P t \cdot W$ and $P t$-Re compounds is not strongly favored. Going to $\operatorname{Ir} P t$, little is to be gained or lost in forming an ordered or cisordered alloy.
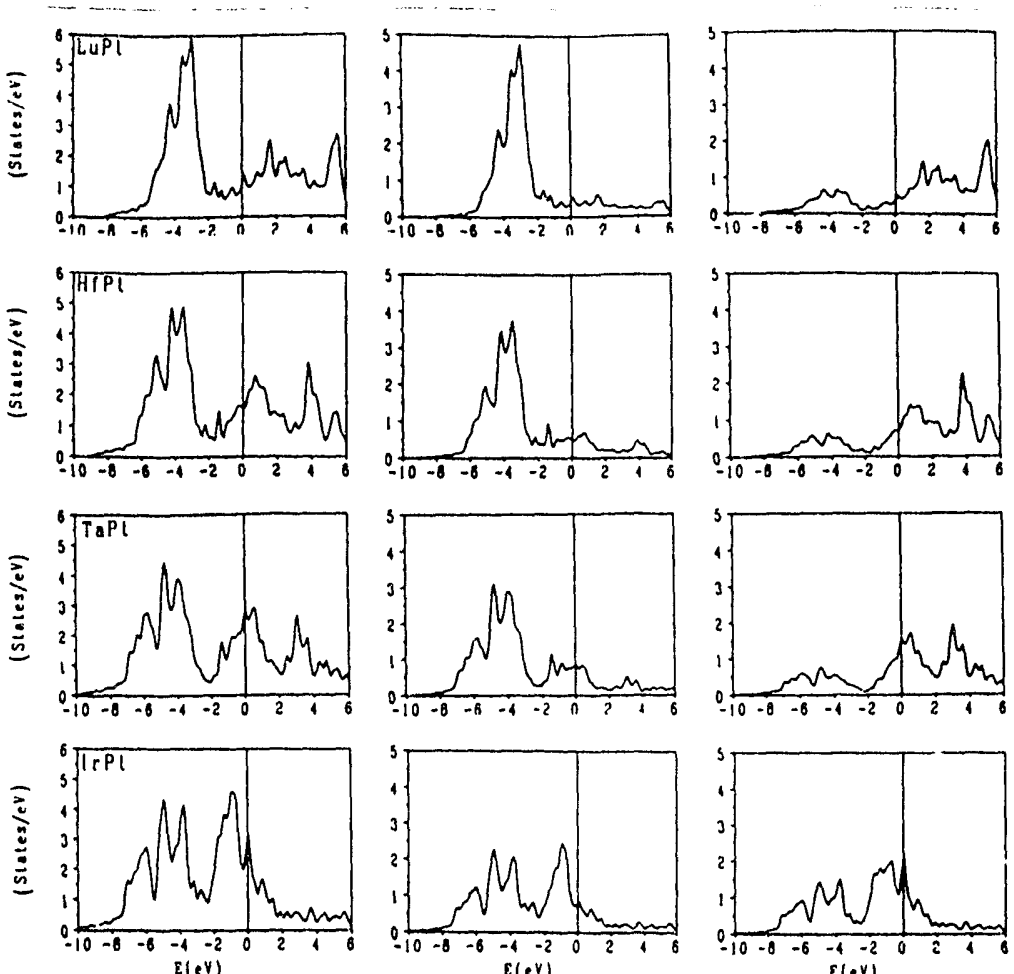

Figure 26. The densities of states for LuPt and threc of the Pt compounds represented in Fig. ure 25 (and 18). The firat column shows the total density of states, the second the density of states localized on the Pt aitea and the third column the local density of states at the site of the other alloy constituent. The zeros of energy of the plots are the Fermi levels. The local densities of states were sampled within the APW spheres and hence do not add up to the total densities of statea.

The heats of formation, calculated ${ }^{35}$ by Schultz and Davenport, for several aluminides appear in Figure 27. These were taken in the $\mathrm{CsCl}$ structure in which all the systems form except $C u A l$, whose structure is more complex. The agreement between theory and experiment is of the order in the spread in experiment. The calculated heats were obtained with full potential calculations though one expects that muffin-tin calculations for these well-packed systems would have done about as well.

In contrast, the calcuiated heats appearing in Figure 5 were obtained with muffin. tin potentials. In general, the calculations are in accord with the experimental heats and the variation in calculated values are consistent with the phase diagram. As a rule, 
those stable phases for which calculations were done, were found to be stable relative phases which are not. The serious exception to this occurs for $R h_{2} r_{2}$ which forms in the $\mathrm{Al}_{2} \mathrm{Cu}$ structure and the muffin-tin calculations have badly underestimated its heat of formation. The $C u$ site in this structue is one of the Bernal-type environments of low coordination, for a metal, and low symmetry. Such a system requires a full potential treatment.

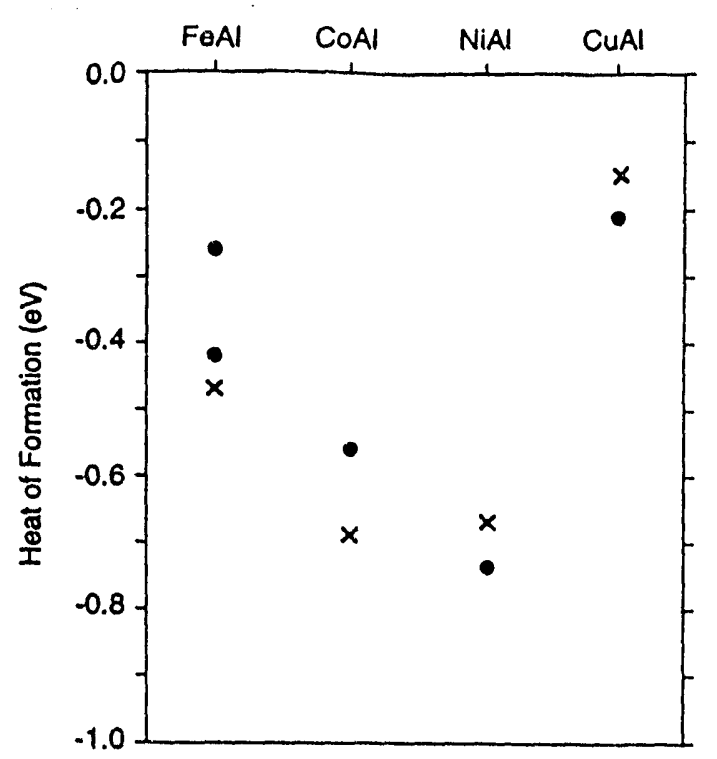

Figure 27. The calculated (x's) and experimental (circles) heats of formation of $F \subset A l, C O A l, N i A l$, and $C$ AAl employing full potential LASTO calculations (binding increases downwards).

The $A u-B f$ and $A u-T a$ systems, which have already been encountered, are represented in Figure 28. These are muffin-tin potentials results ${ }^{30}$ and they are in excellent accord with the experiment values ${ }^{31}$ which came later. The one serious error in the calculation occurs for $\mathrm{AuTa}_{3}$ which forms in the A15 ( $\mathrm{Cr}_{3} \mathrm{Si}$ ) structure, a Frank-Kasper phase, whose heat should be $\sim 0.4 \mathrm{eV} / \mathrm{atom}$ more bound than it is calculated to be, so that it lies at or below the dashed line drawn between $T a$ and $\mathrm{Au}_{2} \mathrm{Ta}_{3}$. As it is, a two phase mix of these two phases is calculated to be more stable than the A15 phase. Again, muffin-tin calculations do well for the well-packed structures and fail for an ill-packed one.

The Pt-Ti system of Figure 2 was chosen for full potential calculations because it" includes one of the two cases, PtTi3, for which a transition metal A15 phase has had its heat measured ${ }^{36}$ calorimetrically. Atomic spheres were chosen for the calculations so that the $T i$ spheres, in the A15 chains, did not overlap. This allowed using the touching atomic spheres of $f c c P t$ to be used for the $P t$ sites. These spheres were then used for all the compounds as well as for the elemental $P t$ and $T_{i}$ reference metals. As has already been noted, the set of calculated $\Delta H$ are in accord with each other and with experiment. Going from a full potential to a muffin-tin treatment reduces the 
binding associated with any single total energy. The cluange in $\Delta H$ between the two treatments depends on whether the full potential is more important for the compound or for the reference elemental metals. The former is the case for PtTij (A15)i

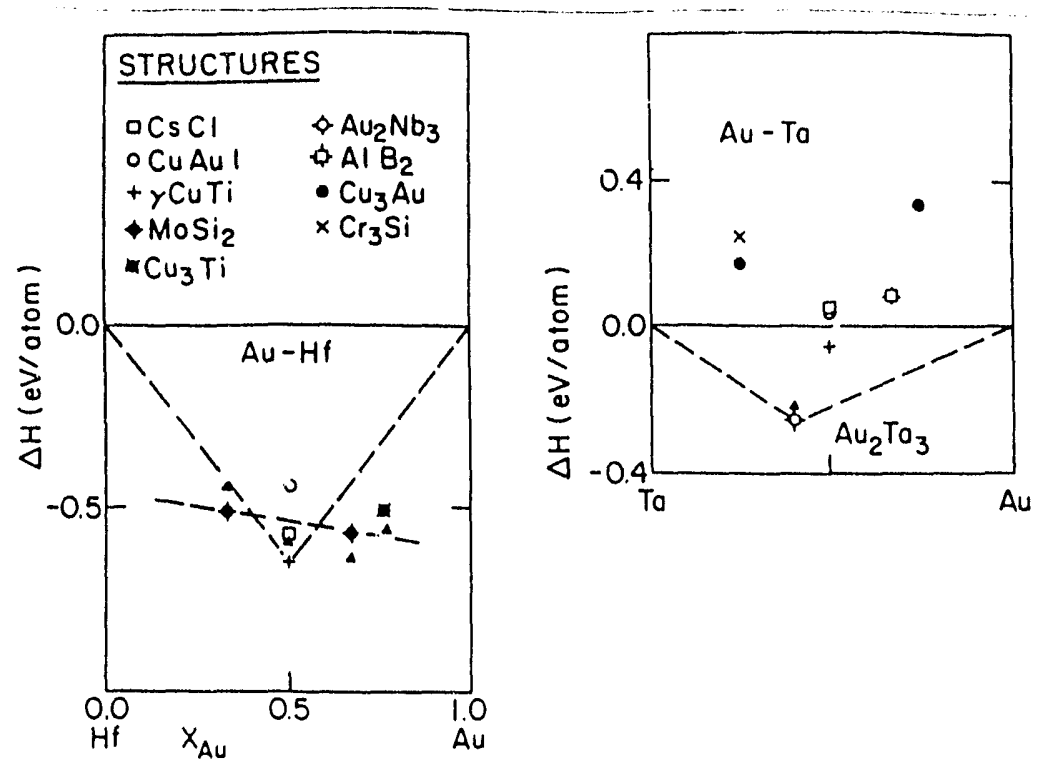

Figure 28. The calculated and experimental (filled trianglea) heats of formation for various phases of $A u-H f$ and Au-Ta for varying alloy componition (binding increases downwards).

it's $\sim 0.15 \mathrm{eV} /$ atom less bound in the muffin-tin treatment. Some of the other phases have their $\Delta H$ more bound, by $\sim 0.1 \mathrm{eV} /$ atom in the muffin-tin scheme. Thus a muffin-tin version of Figure 1 would find the $A 15$ phase less stable, by $1 / 4 \mathrm{eV} /$ atom, relative to the 50:50 phases. The increase in muffin-tin binding for the 50:50 phases is associated with the choice of atomic sphere. The standard choice of atomic spheres for these phases would be to take the touching spheres of the two elemental metals and shrink each equally so that they did not overlap in the compound. Doing this for PtTi $(\mathrm{CsCl}$ ) yields a full potential $\Delta H$ which agrees with the other full potential result (with a shrunken $T i$ and expanded $P t$ ) to better than $0.01 \mathrm{eV} /$ atom. Full potential energies should not, and here are seen not to be, sensitive to sphere choice. The muffin-tin $\Delta H$ for this second sphere choice agrees with its full potential counterpart to $\sim 0.01 \mathrm{eV} /$ atom as well. It would appear that for well-packed structures, atomic sphere choices can be made so that muffin-tin $\Delta H$ are in essential agreement with full potential values. This cannot be done for ill-packed structures.

The skewing of the phase diagram and of the heats of formation, so as to favor the $P t$-rich phases, is readily understood upon inspection of the densities of states plotted in Figure 29. In $P t_{i_{i}}$, the $P t^{\prime}$ 's are far from each other and there is a narrow doublet of primarily $P t 5 d$ character well below the Fermi level. There is a broad majority atom $T i$-like local density of states (LDOS), since $T i$ 's are closer together. The Fermi level falls in these states. Going to the $P t$-rich $\mathrm{Pt}_{3} \mathrm{Ti}_{i}$ and $\mathrm{Pt}_{8} \mathrm{Ti}_{i}$, 
LDOS has become broad and that of the minority $T_{1}$ narrow. At $P t_{3} T_{i}$, the Fermi level falls in the hollow between the "bonding" Pt-like kevels and the "nonbonding" $T i$. Note that the number of bonding and nonbonding states is not equal. The ability to have the bonding levels occupied and the nonbonding empty, favors the $\Delta H$ of $P_{t_{3}} T_{i}$ over, ay, $P t$-poor $P t T_{i j}$. Going to $P t_{8} T i$, the one $T_{i}$ cannot requite the eight platinums and the Fermi level falls in the bonding states with a consequence loss to $\Delta H$. $T i$ contributes enough electrons to the system so that the bonding levels are occupied in $\mathrm{Pt}_{3} \mathrm{Ti}_{\mathrm{i}}$, but it does not have enough for $\mathrm{Pt}_{\mathrm{g}} \mathrm{Ti}_{i}$. For those transition metaltransition metal systems, where the LDOS are separated, as they are in Figure 29, their $\Delta H$ 's are enhanced ${ }^{37}$ when the bonding levels are filled and the nonbonding are empty. Where this happens, of course, depends on the relative numbers of valence electrons of the two constituents.
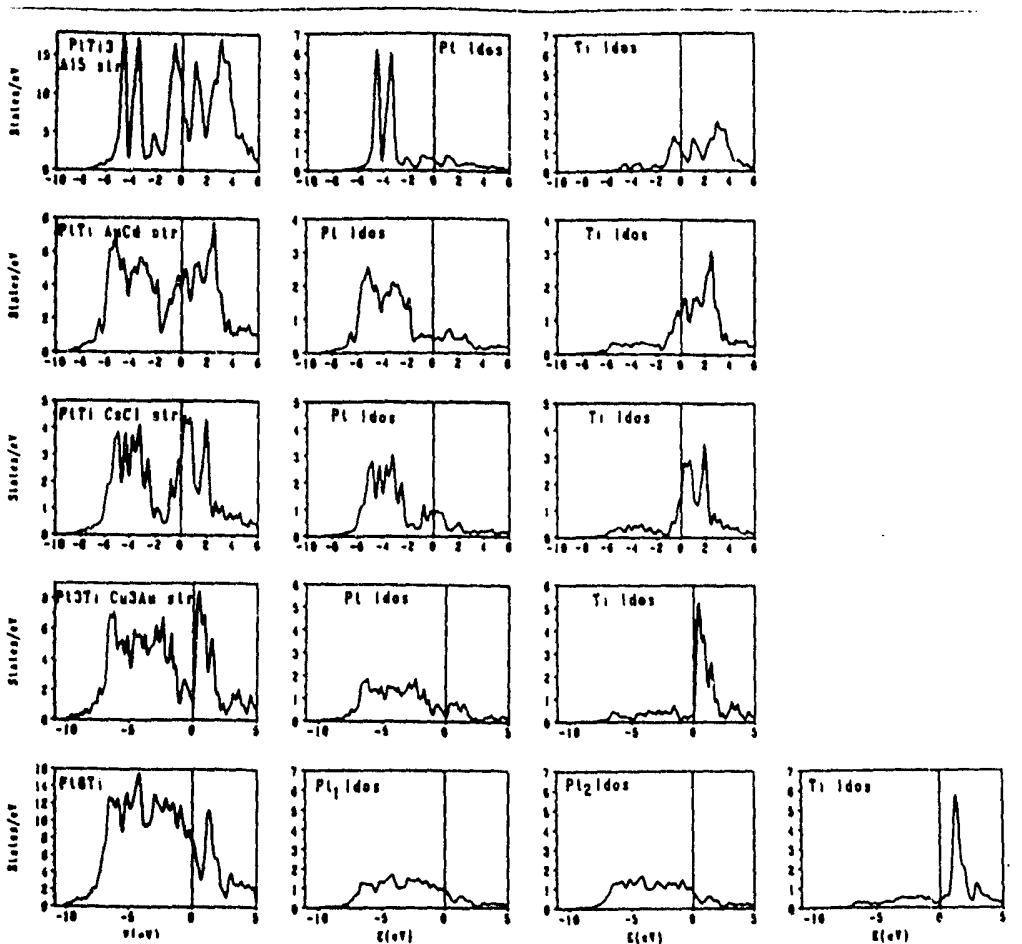

Figare 29. The calculated densitiea of atate (after the manner of Figure 26) for the five PtTi phases represented in Figure 1. The first column shows the lotal densities of states, the other columns beal densities of atates as labeled.

\section{CONCLUSION}

A variety of matters were addressed in this chapter. Let us revisit some of them here. 
Since the early days, there has been concern with charge transfer. In attempting to define it, there is the problem of chosing the atomic volumes to be attributed to the different atomic sites in a compound. A plausible assignment can be made if the deviations from Vegard's law aren't too great, namely use volumes which are propiortional to the elemental volumes. Given such a choice, there remains the question of whether all the charge within some site should be attributed to the atom in that site. As was demonstrated, a significant component of charge is due to tailing, that is the medium associated with the surrounding atoms. While the tailing charge cannot be uniquely defined, it plays a substantial role in the changes in electron counts within atomic site volumes. Examples were shown where, after accounting for charge tailing, the remaining charge transfer was in line with chemical expectations. Experiment shares this difficulty, for it the total charge, whatever its origin, which is sampled by experiment.

Hume-Rothery parameters, such as size and electronegativity, may be used to model alloy stability. They may be used as coordinates in structural maps or employed in effective Hamiltonians, such as Miedema's. Even as "full scale" quantum mechanical calculations become cheaper, such schemes should have their place, both in challenging suspect attributions as to the occurrence of phases and in providing a first pass in predicting the existence of stable and metastable jhases. Such schemes are many orders of magnitude cheaper to implement than any band structure calculation.

Magnetism was considered and it was observed that there are problems with the single determinant character of band theory and with the local density putentials currently used. Nonetheless, spin polarized LSDA calculations have been remarkably successful. The problems become serious when dealing with Hund's second rule orbital magnetic terms. A density dependent potential does not sense the direction of the orbital moment. The aspherical exchange terms of Hartree-Fock theory do have this sense of direction and we propose that the on-site aspherical exchange-correlation terms, within the magnetic shell, of LDA be replaced by their aspherical Hartree-Fock counterpart. This is straightforward for band theory machinery which bas already incorporated the full potential LDA treatment. How well such a resulting spin and orbit polarized magnetic band theory will work, remains to be seen (at least it will avoid the shortcomings of the approximation using the Racah $E^{3}$ or $C$ terms alone).

The original confrontation between band theory and CALPHAD was revisited - namely the issue of the structural energies of the elemental solids. While it can be argued that semiquantit,ative agreement is being approached between the two schools, the disagreement remains serious for those workers constructing phase diagrams via the CALPHAD approach. It seems unlikely that further improvements in band theory estimates will offer a remedy to this matter.

Many alloys form on well-packed $f c c$ or bec lattices where the atomic ordering lowers symmetry causing deviations in lattice positions. Energies of as much as $1 / 3 \mathrm{eV} /$ atom were displayed for such distortions. This appears to be particularly important for layered structures, such as the $\mathrm{MOSi}_{2}$ and $\mathrm{MOPt}_{2}$, and must be dealt with if their stabilities are to be properly estimated.

Many other ordered alloys form in ill-packed structures. One class of these is the Frank-Kasper or tcp (and similarly packed) phases. Another involves atoms in low symmetry, low coordination Bernal-type atomic environments. Both classes of structures appear in transition metal phase diagrams. While muffin-tin potential calculations may suffice for well-packed structures (with results sensitive to the choice of atomic sphere radii), full potentials appear essential to the treatment of the illpacked systems. Any treatment of transition metal alloy phase behavior which claims to be complete, will be confronted with dealing with these systems on an equal footing with the well-packed phases. 
It wiss seen that $d$ band alloy bonding, in the more strongly bonded systems, is maximized when the bonding levels are filled and the nonbonding are empry. In systems displaying reparate peakings of the density of states arising from the separate atomic constituents, the number of bonding and nonbonding levels will not be equal, except at 50:50 alloy concentrations. The optimum filling of bonding states can arise off of 50:50 resulting in the strong skewing seen in the heats of formation and the associated phase diagram as in Figure 1. The compound heats of formation based on LDA calculations and experiment have been seen to agree to $1 / 10 \mathrm{eV} /$ atom which is roughly the scatter in experiment. Although there is little experience as of yet with ill-packed systems, this level of agreement is expected to hold for these as well, providing full potential treatruents are used.

Band theory, as employed, involves an array of approximations. These approxiinations have served us well and they are expected to continue to do so in the future.

\section{ACKNOWLEDGMENTS}

- This work was supported by the Division of Materials Sciences, Office of Basic Energy Scieaces, U.S. Department of Energy under Contract No. DE-AC02. $76 \mathrm{CH} 00016$, and by a grant of computer time at the National Energy Research Supercomputer Center, Livermore, California.

\section{REFERENCES}

1. F.R. deBoer, R. Boom, W.C.M. Matten, A.R. Miedema, and A.K. Niessen. "Cohesion in Metals, Transition Metal Alloya," North Holland, Amsterdam (1989).

2. D.G. Pettifor, J. Phys. C3:367 (1970)

3. R.E. Watson and L.H. Bennett, Scripta Metall. 19:533 (1985).

4. D.R. Nelson, Phys. Rev. B28:5515 (1983).

5. J.D. Bernal, Nature 185:68 (1960)

6. J.D. Bernal, Proc. Roy. Soc. (London) A280:299 (1964).

7. R.E. Wateon and L.H. Bennett, Acto Metall. 32:477 (1984); ibid 32:491 (1984).

8. L.K. Bennett and R.E. Watwon in: "High-Temperature Alloys: Theory and Design, J.O. Stiegler, od., The Metalluryical Society, AIME, Warrendale, PA (1984).

9. R.I. Watson and L.B. Bennett, Phys. Rev, B18:0439 (1978)

10. P. Villars and L.D. Calvert in: "Pearwn's Handbook of Crystallographic Data for Intermetallic Phaces," ASM, Second Edition, Macerials Park, OH (1991)

11. P. Villars, J.Lew-Common Met. 92:215 (1:3); ibid 99:33 (1984); ibid 102:199 (1984); P. Villars and F. Bulliger, J.Lew-Common Met. 132:289 (1987)

12. P. Villars, J.C. Phillips, and H.S. Chen, Phys. Rev. Lett. 57:3085 (1986)

13. K.M. Rabe, A.R. Kortan, J.C. Phillipa, and P. Villara, Phys. Rev. B43:6280 (1991).

14. J.W. Devenport, Phye. Rev. B 29:2896 (1884); G.W. Fernando, J.W. Davenport, R.E. Watson and M. Weinert, ibid B40:2757 (1980).

15. J.P. Perrdew, Physice B172:1 (1991).

16. G. Grimvall, Phyw. Scr. 12:173 (1975)

17. R.E. Watcon and M. Weinert, Phys. Rev. B30:1641 (1984).

18. L. Kaufman and H. Bernatein in: "Computer Calculations of Phase Diagrams," Academic, New York (1870).

19. R.E. Wateon, G.W. Fernando, M. Weinert, YJ. Wang, and J.W. Davenport, Phys. Kev. B43:1455 (1991)

20. O. Gunnaraeon and R.O. Jones, Phys. Rev. B31:7588 (1985); see also J. Harris and R.O. Jones, J. Chem. Phys. 68:3316 (1978)

21. M. Blume and R.E. Watwon, Proc. Roy. Soc. (London) A270:127 (1962).

22. T. Zeigler, A. Reuk, and E.J. Becrends, Theoret. Chim. Acta (Berlin) 43:261 (1977); M. Lan noo, G.A. Aarafi, and M. Schluter, Phys. Rev. B24:943 (1981)

23. U. vonBarth, Phys. Rev. A20:1693 (1979). 
24. J.H. Wood, J. Phys. B13:1 (1980)

25. e.g. O. Eriksson, M.S.S. Brooks, and B. Johansson, J. P'hys Cond Matl 1 4005 (1989); P'hys Rev. B41:9087 (1990).

26. M.R. Norman, Phye. Rev. Lete. 41:7311 (1990); Phyg. Rev, B44:1364 (1991).

27. P.S. Rudman, J. Stinger and R.I. Jafiee, in: "Phase Stability in Metals and Alloya," McGrawBill, New York (1967)

28. G.W. Fernando, R.E. Wateon, M. Weinert, YJ. Wang, and J.W. Davenport, Phys. Rev, $B 41: 11813$ (1990).

29. N. Saunders, A.P. Miodownik, and A.T. Dinedale, CALPHAD 12:351 (1988)

30. R.E. Watwon, J.W. Davenport, and M. Weinert, Phys. Rev. B35:508 (1987)

31. K. Fitzner and O.J. Kleppa, Met. Trans. 23A:997 (1992); K. Fitzner, N. Selhaoui, and O.J. Kleppa, Met. Trans. (in press).

32. R.E. Watson, M. Weinert, and G.W. Fernando, Phys. Rev. B43:1446 (1991)

33. S.S. Jaswa, Y.G. Ren, and D.J. Sellmyer, J. Appl. Phys. 67:4564 (1990)

34. R.E. Wateon, J. Hudis, and M.L. Perlman, Phys. Rev. B4:4139 (1971).

35. P.A. Schultz and J.W. Davenport, Scripla Metall. (in press).

36. N. Selheoui and J.C. Gechon, Analea de Fisica B86:57 (1990)

37. R.E. Wateon, M. Weinert, J.W. Davenport, and G.W. Fernando, Phys. Rev. B39:10,761 (1989). 
DATE FILMED 12123192 
